\title{
Le risque d'attaques de requins à La Réunion
}

Éléments d'analyse des attaques et contextualisation d'une gestion contestée

\section{François Taglioni et Sébastien Guiltat}

\section{OpenEdition}

Journals

Édition électronique

URL : https://journals.openedition.org/echogeo/14205

DOI : 10.4000/echogeo.14205

ISSN : 1963-1197

Éditeur

Pôle de recherche pour l'organisation et la diffusion de l'information géographique (CNRS UMR 8586)

Référence électronique

François Taglioni et Sébastien Guiltat, «Le risque d'attaques de requins à La Réunion », EchoGéo [En ligne], Sur le Vif, mis en ligne le 29 avril 2015, consulté le 22 avril 2022. URL : http://

journals.openedition.org/echogeo/14205; DOI : https://doi.org/10.4000/echogeo.14205

Ce document a été généré automatiquement le 22 avril 2022.

\section{(c) (i) (9)}

EchoGéo est mis à disposition selon les termes de la licence Creative Commons Attribution - Pas d'Utilisation Commerciale - Pas de Modification 4.0 International 


\title{
Le risque d'attaques de requins à La Réunion
}

\author{
Éléments d'analyse des attaques et contextualisation d'une gestion \\ contestée
}

\author{
François Taglioni et Sébastien Guiltat
}

\section{Introduction}

1 En dépit de leur rôle primordial sur l'équilibre des écosystèmes marins, les requins restent au centre d'une hésitation des pouvoirs publics entre leur nécessaire préservation et le maintien des activités nautiques de loisirs. La gestion du risque d'attaque du requin, couramment dénommé "risque requin», est motivée par la relative augmentation du nombre mondial d'attaques depuis quelques décennies. L'île de La Réunion n'y échappe pas ; la dernière attaque mortelle en date s'est produite sur un surfeur de 13 ans le dimanche 12 avril 2015, après une série de 16 attaques dont 6 mortelles entre 2011 et février 2015. On peut cependant considérer que le risque d'attaque du requin pour la population réunionnaise et les touristes qui séjournent dans l'île est beaucoup plus faible que bien d'autres risques présents sur le territoire.

Ce risque a déjà fait couler beaucoup d'encre dans les médias régionaux, nationaux et internationaux. Et ceci à tel point que ces derniers l'ont qualifié de " crise du requin ». Le terme «crise" s'entend ici par les conséquences majeures des attaques de requins tant au niveau humain, avec un nombre croissant de victimes ces dernières années, qu'en terme d'impact économique, social et politique (Fabing, 2014 ; Jaccoud, 2014). On est sans doute loin de l'acception plus classique de la notion de crise qui s'entend par une dégradation des rétroactions d'un système aboutissant à une situation d'incertitude de quelque temps, puis à la mise en place de nouvelles rétroactions voire d'un nouvel équilibre. On peut également considérer que «crise signifie indécision : c'est le moment où, en même temps qu'une perturbation, surgissent les incertitudes » (Libaert, 2009) et de ce point de vue, la gestion de la crise du requin est éclairante. 
3 À ce jour, les attaques de requins, comme objet d'analyses socio-spatiales, n'ont que peu été investies par les chercheurs en sciences sociales et humaines. Pour essayer de contribuer à une réflexion scientifique à propos d'une préoccupation majeure pour le développement territorial de La Réunion, cet article comporte d'une part une analyse de données sur les attaques depuis 1980 et, d'autre part, une réflexion sur la gestion institutionnelle de la crise du requin qui affecte La Réunion depuis 2011. Quelle est la place de La Réunion dans la hiérarchie mondiale des attaques? Comment se distribuent-elles sur le territoire réunionnais? Quels sont les facteurs de vulnérabilité, les enjeux et les éléments explicatifs de ces attaques pour les populations et la société locale? En quoi l'ampleur prise par cette crise est-elle révélatrice d'un dysfonctionnement de la gouvernance et des modes de gestion de crise à La Réunion? Dans quelle mesure une gestion anticipée de ce risque en aurait-elle diminué les impacts? Autant de questions que soulève cette succession hors-norme d'attaques sur un territoire exigu et à la vulnérabilité économique et sociale structurelle marquée (Roinsard, 2014).

\section{L'analyse du risque d'attaques de requins}

\section{La Réunion dans le risque mondial d'attaques de requins}

Nous avons retenu comme définition des attaques de requins une agression provoquée ou non d'un requin (ou de requins) sur une personne humaine vivante, qui occasionne des blessures, voire le décès de la victime ou encore une détérioration de son équipement (planche de surf, planche de bodyboard, planche à voile, planche de kite surf, kayak, pirogue, palmes... etc). Une définition plus restrictive ne prend en compte que les interactions entre un requin et une personne vivante sans tenir compte de la simple détérioration de son équipement ou d'une attaque de défense (Rard, 2013) ou alors excluant les dégradations d'embarcations, les attaques sans morsure et les prédations posthumes (Van Grevelynghe et alii, 1999). Une autre prend aussi en considération les interactions entre un requin et une personne déjà décédée avant l'attaque et qui doit obligatoirement comporter une morsure (Burgess, Callahan, 1996).

5 À l'échelle mondiale, on peut constater que le risque requin a augmenté ces dernières décennies. De quelques attaques (illustration 1) répertoriées au début des années 1980, on dépasse la centaine annuelle depuis les années 2000 , avec une relative régularité du nombre des attaques mortelles qui s'établissent autour de 10 par an en moyenne. Ces premières données extraites des fichiers de compilations Shark Attack Survivors ${ }^{1}$ sont fiables et ont été améliorées au fil des années. Elles comportent cependant des biais de collecte et de méthodologie qui les rendent perfectibles notamment pour les décennies 1980 et 1990. D'autre part, il faut considérer que la population mondiale n'a pas cessé d'augmenter depuis 1980 (avec un gain de près de 3 milliards d'habitants) et que, dans le même temps le nombre de pratiquants de sports côtiers a lui-même considérablement augmenté avec la croissance des niveaux de vie, la littoralisation de la population mondiale, l'apparition de nouvelles activités de loisir (kite surf, stand up paddle, kayak de mer, wake board, planche à voile ...) et de nouveaux matériaux permettant de les pratiquer toute l'année. A contrario, la population mondiale des requins diminue constamment (Worm et alii, 2012) avec une estimation de prélèvements annuels de l'ordre 100 millions d'individus en 2010 (Worm et alii, 2012). 
Illustration 1 - Évolution des attaques de requins dans le monde entre 1980 et 2013

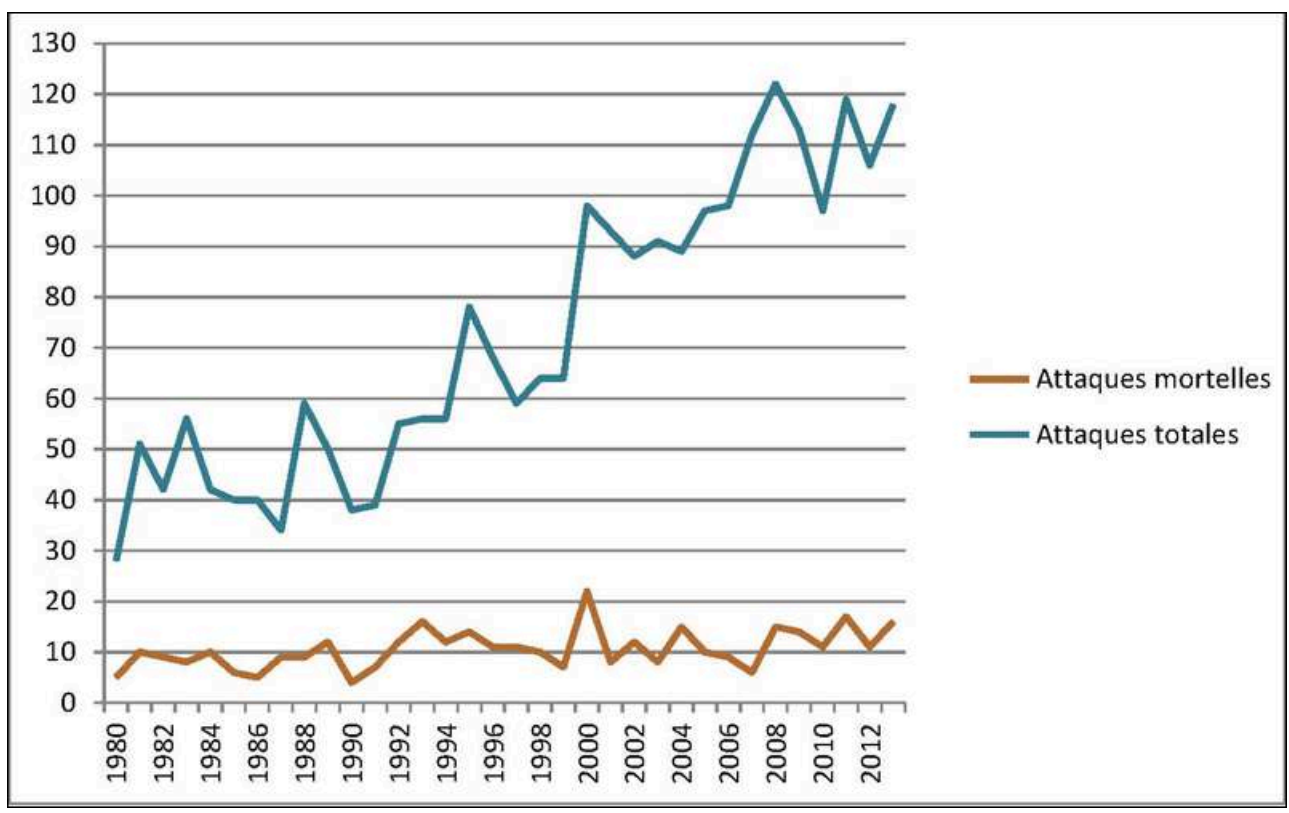

Source : Shark attack file info (http://sharkattackfile.info).

6 Si l'on compare maintenant l'évolution des attaques de requins dans le monde et à La Réunion depuis 1980 (tableau 1), on remarque que La Réunion a été le théâtre en moyenne de $2 \%$ des attaques annuelles mondiales et de $6 \%$ des attaques annuelles mortelles. Eu égard à la population de La Réunion (840 000 habitants en 2013), on peut déjà affirmer que ces deux moyennes placent La Réunion en position de surreprésentation d'attaques et plus encore d'attaques mortelles.

Tableau 1- Évolution moyenne des attaques de requins dans le monde entre 1980 et 2013

\begin{tabular}{|l|l|lr|lr|}
\hline $\begin{array}{l}\text { Nombre moyen } \\
\text { d'attaques annuelles } \\
\text { dans le monde } \\
1980-2013\end{array}$ & $\begin{array}{l}\text { Nombre moyen } \\
\text { d'attaques annuelles } \\
\text { à La Réunion } \\
1980-2013\end{array}$ & $\begin{array}{l}\text { Nombrerayen } \\
\text { d'attaques mortelles } \\
\text { annuelles dans le monde } \\
1980-2013\end{array}$ & $\begin{array}{l}\text { Nombrer moyen } \\
\text { d'attaques rertelles } \\
\text { annuelles à La Réunion } \\
1980-2013\end{array}$ \\
\hline 72 & 1,3 & 10 & 0,6 \\
\hline
\end{tabular}

Sources : Shark attack file info (http://sharkattackfile.info) ; sources de l'illustration 2 pour La Réunion.

7 Pour affiner ces premières analyses, quelques indicateurs relatifs de la place de La Réunion dans le classement mondial des attaques sont nécessaires. Nous avons retenu comme période de référence pour les tableaux qui suivent (tableaux $2 \mathrm{a}$ et $2 \mathrm{~b}$ ) celle qui s'étend de 2011 à 2014, car elle correspond à ce que l'on a nommé la " crise du requin » à La Réunion. Notre choix, en matière d'espaces d'analyses, est celui des territoires les plus concernés dans le monde par les attaques de requins. Il s'agit, par ordre décroissant, des États-Unis (avec deux États particulièrement concernés que sont la Floride et Hawaï), de l'Australie, de la République d'Afrique du Sud et de La Réunion. Sur la période considérée, ces États et territoires à eux seuls, concentrent plus des trois-quarts (76\%) des attaques mondiales dont près de la moitié (47 \%) aux États-Unis. 
Pour la part des attaques mortelles, la hiérarchie est modifiée, l'Australie étant en tête suivie de la République d'Afrique du sud, de La Réunion et des États-Unis d'Amérique. La proportion d'attaques mortelles dans les attaques totales place La Réunion en tête de ce classement avec une attaque mortelle sur 3. Il en va de même, et de très loin, pour presque tous les autres indicateurs que sont le nombre d'attaques mortelles ou non pour un million d'habitants et le nombre d'attaques mortelles ou non pour $100 \mathrm{~km}$ de linéaire côtier.

Tableau 2a - Données sur les attaques dans le monde en comparaison de La Réunion depuis la crise du requin de 2011 (Janvier 2011 à septembre 2014)

\begin{tabular}{|c|c|c|c|c|c|}
\hline & $\begin{array}{l}\text { Nombre } \\
\text { d'attaques } \\
\text { Valeur } \\
\text { absolue }\end{array}$ & $\begin{array}{l}\text { Part } \\
\text { mondiale des } \\
\text { attaques } \\
\%\end{array}$ & $\begin{array}{l}\text { Nombre } \\
\text { d'attaques } \\
\text { mortelles } \\
\text { Valeur absolue }\end{array}$ & $\begin{array}{l}\text { Part mondiale des } \\
\text { attaques mortelles } \\
\%\end{array}$ & $\begin{array}{l}\text { Part des } \\
\text { attaques } \\
\text { mortelles } \\
\text { dans les } \\
\text { attaques } \\
\text { totales } \\
\%\end{array}$ \\
\hline États-Unis (total) & 193 & 47 & 4 & 8,5 & 2 \\
\hline - Floride & 88 & 21 & 1 & 2 & 1,1 \\
\hline - Hawaï & 34 & 8 & 2 & 4 & 6 \\
\hline Australie & 74 & 18 & 13 & 26,5 & 17,6 \\
\hline $\begin{array}{l}\text { Rép. } \\
\text { d'Afrique du Sud }\end{array}$ & 28 & 7 & 8 & 16 & 28,6 \\
\hline La Réunion & 15 & 4 & 5 & 10 & 33 \\
\hline $\begin{array}{l}\text { Part de ces } 4 \text { pays } \\
\text { en } \% \text { du } \\
\text { mondial }\end{array}$ & / & 76 & / & 61 & \\
\hline
\end{tabular}

Sources : Shark attack file info (http://sharkattackfile.info) ; sources de l'illustration 2 pour La Réunion ; méthodologie et calcul d'Olivier Hoffer (2013) pour la mesure précise des linéaires côtiers.

Tableau $2 \mathrm{~b}$ - Données sur les attaques dans le monde en comparaison de La Réunion depuis la crise du requin de 2011 (Janvier 2011 à septembre 2014)

\begin{tabular}{|c|c|c|c|c|}
\hline & $\begin{array}{l}\text { Nombre d'attaques } \\
\text { pour un million } \\
\text { d'habitants }\end{array}$ & $\begin{array}{l}\text { Nombre d'attaques } \\
\text { mortelles pour un } \\
\text { million d'habitants }\end{array}$ & $\begin{array}{l}\text { Nombre } \\
\text { d'attaques } \\
\text { pour } 100 \mathrm{~km} \\
\text { de } \quad \text { linéaire } \\
\text { côtier }\end{array}$ & $\begin{array}{l}\text { Nombre } \\
\text { d'attaques } \\
\text { mortelles pour } \\
100 \quad \mathrm{~km} \quad \mathrm{de} \\
\text { linéaire côtier }\end{array}$ \\
\hline
\end{tabular}




\begin{tabular}{|l|l|l|l|l|}
\hline $\begin{array}{l}\text { États-Unis } \\
\text { (total) }\end{array}$ & 0,06 & 0,01 & 1,7 & 0,03 \\
\hline - Floride & 4,63 & 0,05 & 4,3 & 0,05 \\
\hline - Hawaï & 24,28 & 1,43 & 2,6 & 0,15 \\
\hline Australie & 3,22 & 0,56 & 0,5 & 0,08 \\
\hline Rép. & 0,54 & 0,15 & 1,1 & 0,29 \\
d'Afrique du Sud & $\mathbf{1 7 , 0 4}$ & $\mathbf{5 , 6 8}$ & $\mathbf{7 , 1}$ & $\mathbf{2 , 4}$ \\
\hline $\begin{array}{l}\text { La Réunion } \\
\text { Part de ces 4 } \\
\text { pays en du } \\
\text { total mondial }\end{array}$ & & & & \\
\hline
\end{tabular}

Sources : Shark attack file info (http://sharkattackfile.info) ; sources de l'illustration 2 pour La Réunion ; méthodologie et calcul d'Olivier Hoffer (2013) pour la mesure précise des linéaires côtiers.

8 On voit à nouveau que La Réunion, petit espace insulaire de $2512 \mathrm{~km}^{2}$, peuplé de 840000 habitants, présente une vulnérabilité face au risque requin (et spécialement face aux attaques mortelles) qui est globalement très supérieure à celle des autres territoires concernés dans le monde et ceci particulièrement depuis 2011. Ce constat demande à être approfondi par des analyses plus fines à l'échelle de La Réunion où la répartition des attaques n'est pas homogène sur l'ensemble du territoire et renforce ainsi, nous allons le voir, non seulement la position première de l'île dans la hiérarchie mondiale mais aussi génère une véritable limite à ses dynamiques socio-spatiales.

\section{Une surreprésentation des attaques dans la zone balnéaire de l'ouest de La Réunion}

9 Pour pouvoir aller plus loin dans les analyses et construire la carte interactive (illustration 2), nous avons constitué une base de données sur les attaques de requins depuis 1980 à La Réunion. Cette base est fondée sur les compilations déjà citées de la Shark Attack Survivors ${ }^{2}$ qui font aujourd'hui référence parmi les nombreuses bases mondiales sur les attaques de requins. Néanmoins, elle n'est pas exhaustive et est parfois entachée d'erreurs. Nous l'avons donc complétée, amendée et corrigée à partir d'un travail de compilation méticuleux qui s'appuie d'une part sur le dépouillement de la presse locale (La Réunion) et nationale (France métropolitaine) et d'autre part sur la comparaison avec des bases de données locales sur les attaques de requins et des entretiens avec les acteurs locaux impliqués dans le suivi de ces attaques ${ }^{3}$.

La série retenue pour notre base de données comporte les 46 attaques, dont 20 furent mortelles, qui se sont produites entre le 24 novembre 1980 et le 22 juillet 2014. Les données antérieures à 1980 se sont révélées trop approximatives et sans doute incomplètes pour être exploitables. Enfin, les 2 attaques mortelles des 14 février 2015 et 12 avril 2015, bien qu'incluses dans la carte interactive, ne sont pas prises en compte 
dans toutes les analyses qui vont suivre car elles interviennent après que cet article ait été scientifiquement validé pour publication.

11 Nous avons intégré dans la base, pour chaque attaque, les données suivantes :

- la date de l'attaque

- le lieu de l'attaque

- l'heure, parfois approximative, de l'attaque

- l'activité de la victime au moment de l'attaque. Ces activités ont été réparties en cinq catégories :

- sports de glisse (surf, bodyboard, planche à voile, kayak de mer, pirogue)

- chasse sous-marine

- nage

- plongée en bouteilles

- autres (2 attaques, l'une concernant un pêcheur de bichiques ${ }^{4}$ et une deuxième dont l'activité de la victime était inconnue au moment de l'attaque mortelle qu'elle a subie)

- la gravité de l'attaque, mortelle ou non

- le type de requin impliqué dans l'attaque (cette donnée est très souvent incomplète ; elle n'est donc pas exploitée directement dans l'illustration 2), mais il est établi que les requins bouledogues (Carcharhinus leucas) et plus marginalement les requins tigres (Galeocerdo cuvier) sont les principaux protagonistes pour les données disponibles à ce sujet (Gauthier, 2012).

Illustration 2 - Les attaques de requins à La Réunion depuis 1980 (Données au 20 avril 2015)

[cliquer sur ce lien pour accéder à la carte]

La carte interactive sur les attaques de requins à La Réunion depuis 1980 (illustration 2) est la seule produite à ce jour sur le sujet. Elle permet d'avoir une vue d'ensemble exhaustive sur la répartition spatiale des attaques, de connaître la gravité de l'attaque, le type d'activité pratiquée au moment de l'attaque, le lieu, la date et l'heure ainsi qu'un filtrage par année.

carte nous permet en outre de développer des analyses sur la répartition spatiale des attaques, sur l'activité des victimes au moment de l'attaque, sur la spécificité des attaques mortelles, sur le jour, l'heure, la saisonnalité et l'annualité des attaques.

La répartition spatiale des attaques (illustration 2) fait très nettement apparaître une dissymétrie est/ouest suivant une ligne allant de la commune du Port (Piscine) à la commune de Saint-Joseph (Cap Méchant). La partie occidentale concentre en effet plus des trois-quarts des attaques ( 36 sur les 46 ) durant la période considérée qui s'étale de 1980 à 2014. Plus particulièrement, ce sont les plages de la baie de Saint-Paul, de Boucan Canot, des Roches Noires, de Trois Bassins, de Saint-Leu, de l'Etang Salé, de Saint-Pierre (Pic du Diable) et de Petite-Ile qui forment les principaux secteurs d'attaques. Cette dissymétrie s'explique, d'une part par la forte concentration de la population réunionnaise dans la partie occidentale de l'île où vivent $70 \%$ de la population totale. D'autre part, l'existence et la fréquentation des «spots » de sports côtiers sont des éléments déterminants de cette répartition. En effet, les "spots ${ }^{5}$ » de sports de glisse, de plongée en bouteilles et de sites de baignade sont presque exclusivement situés dans cette partie de l'île (Bessy, Naria, 2004). La situation pour les sites de chasse sous-marine est un peu différente puisque, bien que répartis de façon assez homogène sur l'île, les pratiquants se trouvent très majoritairement (Rard, Menou, 2011) dans la partie orientale de l'île, allant des communes de Saint-Philippe à 
Saint-Denis. Ceci explique sans doute que 2 des 10 attaques qui se sont déroulées à l'est le furent sur des chasseurs sous-marins. Les principaux sites d'attaques dans l'est se situent autour de Saint-Denis et de Saint-Benoît avec une absence d'attaques sur le littoral du volcan enclavé, un endroit peu accessible aux pratiquants. Néanmoins, in fine, la partie occidentale de l'île, telle que nous l'avons définie, concentre de l'ordre de 90 \% (Rard, Menou, 2011) de la fréquentation du littoral réunionnais

Plus précisément, en ce qui concerne l'activité des victimes au moment des attaques, les pratiquants les plus touchés (illustration 3) sont ceux qui s'adonnent aux sports de glisse avec presque les deux tiers (61\%) du total, soit 28 des 46 attaques. Parmi ces pratiquants, les surfeurs sont les plus représentés suivis des bodyboardeurs et dans une moindre mesure des véliplanchistes et de la catégorie " autres » (pirogue et kayak, une attaque pour chacune de ces deux activités). Cette proportion d'attaques au sein des activités de sports de glisse est à mettre en perspective avec le nombre majoritaire de pratiquants de ces sports parmi les usagers du littoral de la côte occidentale (Guiltat, 2011).

Illustration 3 - Part (\%) des attaques par type d'activité depuis 1980

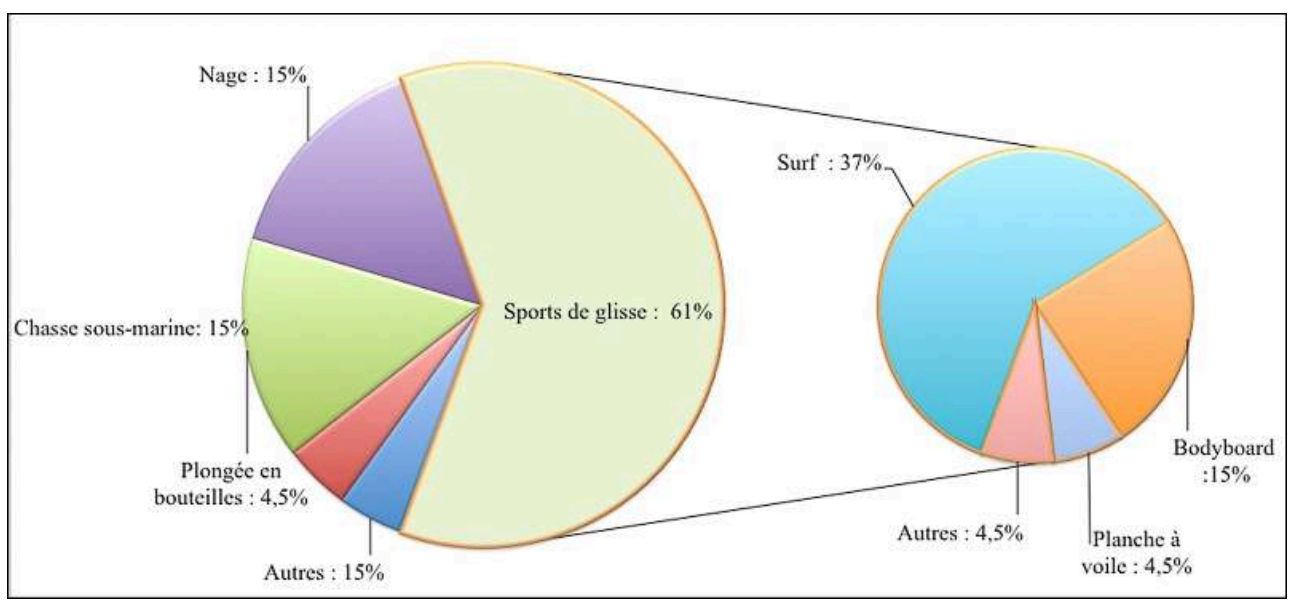

Sources : celles de l'illustration 2.

Viennent ensuite à part égale (15\%) les nageurs et les chasseurs sous-marins qui sont vulnérables face aux attaques quelle que soit leur distance du rivage (l'attaque du 15 juillet 2013 dans la baie de Saint-Paul est significative à ce sujet, puisque la victime avait pied et se trouvait très près du rivage). Les plongeurs en bouteilles sont les moins vulnérables en dépit du nombre estimé élevé de pratiquants (Guiltat, 2011). La catégorie "autres » couvre 2 attaques (pêcheur de bichiques et activité inconnue au moment de l'attaque) est difficile à discuter en raison d'un nombre peu significatif de victimes.

Nous l'avons dit, l'ouest de l'île est le théâtre majeur des attaques depuis 1980. Cette côte est en partie (sur environ la moitié du littoral de la zone ouest) occupée par la Réserve naturelle marine de La Réunion (RNMR, cf. illustration 2) ${ }^{6}$. En 26 ans, entre 1980 et 2006, 7 attaques avaient eu lieu dans le futur périmètre de la RNMR, dont 2 mortelles, soit une moyenne de 0,3 attaques par an. Depuis 2007 (date de l'ouverture de la RNMR), ce sont 11 attaques dont 4 mortelles qui se sont produites en moins de 8 ans ; soit une moyenne de 1,5 attaques par an. Dans le même intervalle, entre 1980 et 2006, on dénombre 22 attaques hors RNMR, dont 14 mortelles (soit une moyenne de 
0,9 attaques par an) et entre 2007 et 2014 ce sont 5 attaques hors RNMR, dont 1 mortelle (soit une moyenne de 0,6 attaques par an) qui ont été recensées. Il apparait ainsi (tableau 3) que le nombre moyen annuel d'attaques dans la RNMR a été multiplié par 5 depuis 2007 et que cette moyenne annuelle hors RNMR a été divisée par 1,5 depuis la même date. Cette impression de concentration des attaques dans la RNMR est accentuée par le fait que sur les 5 attaques mortelles depuis 2007, 4 se sont déroulées dans ce périmètre.

Tableau 3 - Nombre moyen annuel d'attaques de requins à La Réunion depuis 1980 dans la RNMR et hors RNMR

\begin{tabular}{|l|l|l|}
\hline & $\begin{array}{l}\text { Nombre moyen annuel } \\
\text { d'attaques dans la RNMR }\end{array}$ & Nombre moyen annuel d'attaques hors RNMR \\
\hline Entre 1980 et 2006 & $0,3 /$ an (7 attaques en 26 ans) & $0,9 /$ an (22 attaques en 26 ans) \\
\hline Depuis 2007 & $1,5 /$ an (12 attaques en 8 ans) & $0,6 /$ an (5 attaques en 8 ans)) \\
\hline Total 1980-2014 & $0,6 /$ an (19 attaques en 35 ans) & $0,8 /$ an (27 attaques en 35 ans) \\
\hline Depuis 2011 & $2,2 /$ an (11 attaques en 5 ans) & $0,8 /$ an (4 attaques en 5 ans) \\
\hline
\end{tabular}

Sources : celles de l'illustration 2. contemporaine du requin (2011-2014), sur 15 attaques on en dénombre alors 11 dans la
RNMR dont 4 mortelles. Sur cette période, la part des attaques dans la RNMR est donc de près de $75 \%$ du total et celle des attaques mortelles de $80 \%$. Sachant que la RNMR présente un linéaire côtier de $40 \mathrm{~km}$, le nombre d'attaques pour $100 \mathrm{~km}$ de linéaire côtier passe donc de 7,1 (tableau 2b) pour l'ensemble de La Réunion ( $210 \mathrm{~km}$ de linéaire côtier) à $27,5^{7}$ dans la RNMR et le nombre d'attaques mortelles par km de linéaire côtier passe de 2,4 à 10. La RNMR devient ainsi, bien que les données ne soient pas standardisées, sans doute l'une des zones les plus risquées du monde depuis $2011^{8}$. On comprend mieux l'émoi suscité par ces attaques depuis 2011 auprès des pratiquants de sports côtiers dans la RNMR. D'autant plus que, si l'on considère que les enjeux sont "ce que l'on peut perdre » (Metzger; D’Ercole, 2011), quand on sait que la moyenne d'âge de l'ensemble des victimes d'attaques depuis 1980, tous de sexe masculin (sauf une victime), est de 29 ans $^{9}$ (Gauthier, 2012), on ne peut être que profondément frappé par ces attaques. D'autant, qu'elles sont très relayées médiatiquement tant sur le plan local que national avec en toile de fond sans doute une amplification des accidents liée à une peur spécifique (effet «Les dents de la mer ", Neff, 2014 et Curmi, 2005). Les données statistiques montrent que le risque d'attaques de requins reste très faible comparé à toutes les autres causes possibles d'accidents dans le milieu marin et plus encore terrestre.

Peut-on pour autant, lier directement l'augmentation moyenne des attaques dans la RNMR au fait que cette aire protégée soit devenue poissonneuse au point d'attirer les requins? Selon les conclusions d'une étude récente (Bigot et alii, 2014), il apparaît que « la biomasse en poissons des récifs de La Réunion reste encore faible, très loin de pouvoir déjà traduire de manière objective et avérée un "effet réserve significatif » 
(illustration 7). Par ailleurs, la RNMR accueille à elle seule environ $80 \%$ des utilisateurs du littoral de La Réunion et des pratiquants de sports côtiers (Rard; Menou, 2011). Cette forte concentration des pratiquants fournit déjà une bonne explication aux 19 attaques sur 46 recensées depuis 1980. Par ailleurs, si nous revenons à la série de données de 1980 à 2014 (illustration 2), on peut noter qu'il y a déjà eu des "séries noires » d'attaques de requins ces dernières décennies à La Réunion. Par exemple, entre le 22 mai 1992 et le 11 avril 1999, soit en moins de 7 ans, on dénombre 12 attaques dont 10 mortelles réparties aux quatre coins de l'île.

21 L'analyse de la part des attaques mortelles par type d'activité depuis 1980 (illustration 4) apporte un éclairage sur la vulnérabilité des pratiquants. Sur les 46 attaques enregistrées dans la base entre 1980 et 2014, 20 ont été mortelles. Le taux de mortalité est donc de $43 \%$, ce qui place La Réunion bien au-dessus de la moyenne mondiale qui s'établit d'après nos calculs ${ }^{10}$, sur la même période, à $14 \%$. Cette mortalité exceptionnelle est sans doute imputable pour partie au type de requins impliqués dans les attaques à La Réunion [requins bouledogues et plus marginalement requins tigres, tous deux macrophages (Gauthier, 2012)]. Les pratiquants de sports de glisse représentent la moitié des victimes d'attaques mortelles (illustration 4). Dans cette catégorie, les surfeurs sont en première ligne, suivis des bodyboardeurs et des véliplanchistes. Cette hiérarchie au sein des sports de glisse est sans doute conforme au nombre estimé de pratiquants de chaque activité (Guiltat, 2011). En revanche, en terme de vulnérabilité (illustration 5), il apparaitt très nettement que les bodyboardeurs sont plus exposés que les surfeurs avec un taux de mortalité de $43 \%$ (3 attaques mortelles sur les 7 attaques) alors que les surfeurs présentent un taux de mortalité de $29 \%$ (5 attaques mortelles sur les 17 attaques). Le fait que les bodyboadeurs aient davantage le corps immergé dans l'eau durant une session de glisse constitue probablement une première explication à cette plus grande vulnérabilité. D'autre part, les surfeurs peuvent plus facilement utiliser leur planche pour se protéger lors des attaques par rapport aux bodyboardeurs qui ont une planche beaucoup plus courte. D'ailleurs pour quelques attaques sur des surfeurs, la seule interaction a été limitée à des dégâts sur le flotteur et pas sur le pratiquant. C'est moins le cas pour les bodyboardeurs. Pour les véliplanchistes, bien que le taux de mortalité soit de $100 \%$, il n'est pas possible d'en tirer des conclusions car l'échantillon des attaques est limité à 2 et l'on ne connaît pas la position des victimes par rapport à leur planche au moment des attaques. Peut-être étaient-ils dans la même situation qu'un nageur, loin de leur planche lors de l'attaque. Les nageurs constituent en effet une catégorie très vulnérable. Ils représentent $30 \%$ des attaques mortelles (illustration 4) mais leur taux de mortalité est de 85\% (6 attaques mortelles sur les 7 attaques recensées (illustration 5)). Ils n'ont en effet aucun moyen de protection au moment de l'attaque. C'est d'ailleurs la catégorie la plus vulnérable si l'on émet l'hypothèse que les 2 attaques sur des véliplanchistes étaient assimilables à des attaques sur des nageurs. Plongée en bouteilles et chasse sous-marine constituent à part égale $5 \%$ des attaques mortelles (illustration 4), mais la vulnérabilité des plongeurs en bouteilles semble plus élevée ( $50 \%$, illustration 5 ) que celle des chasseurs sousmarins (14\%). Dans les faits, il est difficile d'estimer de façon réaliste la vulnérabilité des plongeurs en bouteilles puisque le nombre d'attaques est de 2, dont une mortelle. En revanche, pour les chasseurs sous-marins, le nombre d'attaques est de 7 dont 1 mortelle, ce qui est statistiquement plus significatif. Au final, les plongeurs, qu'ils soient en bouteilles ou chasseurs, sont sans doute les moins vulnérables face au risque requin. Enfin, la catégorie « autres» (10\% des attaques mortelles, illustration 4$)$ n'est 
pas exploitable car elle concerne 2 attaques (toutes deux mortelles) dont l'une est unique en son genre (pêcheur de bichiques) et l'autre aux conditions inconnues.

Illustration 4 - Part (\%) des attaques mortelles par type d'activité depuis 1980

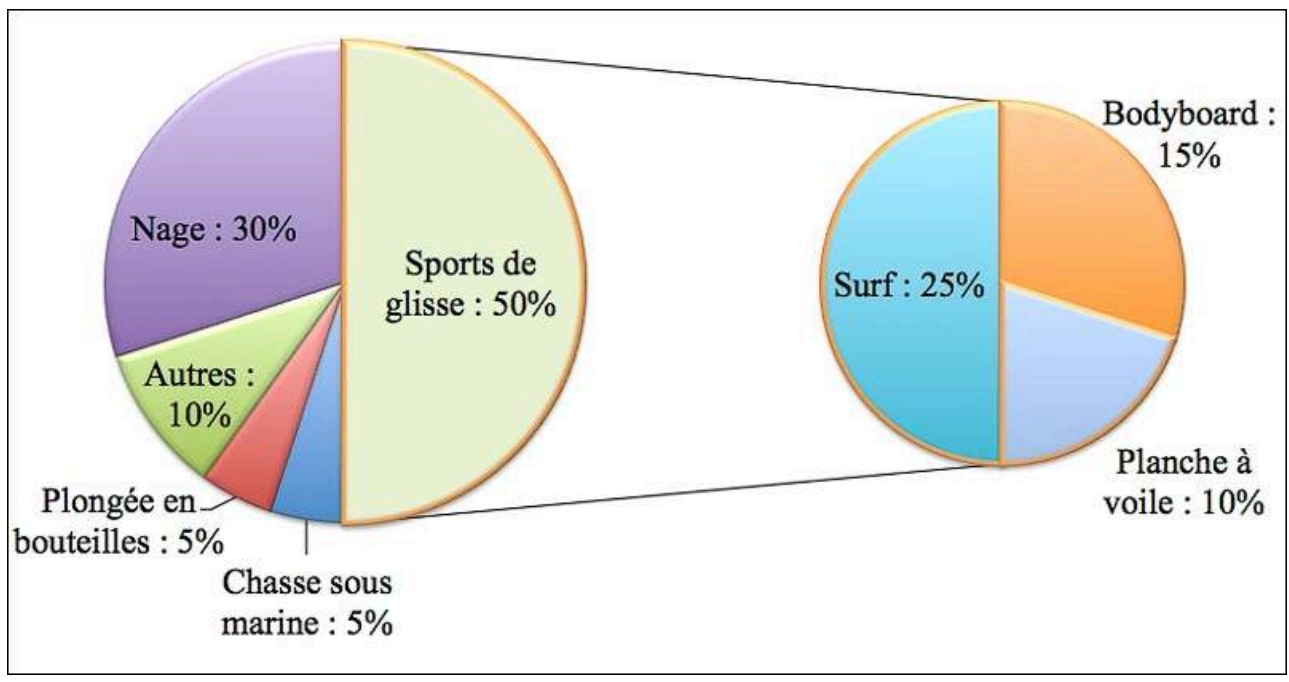

Sources : celles de l'illustration 2.

Illustration 5 - Taux de mortalité (\%) des attaques par type d'activité

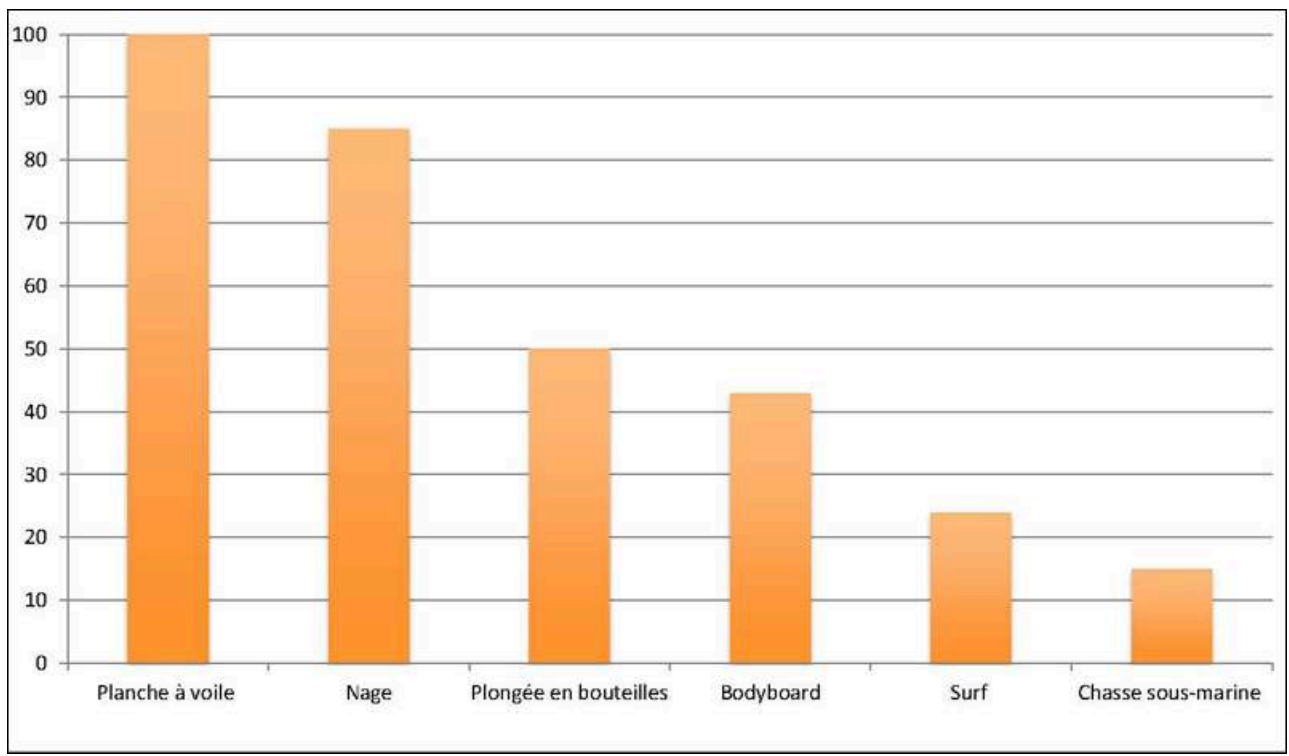

Sources : celles de l'illustration 2.

Un rapide examen de la répartition des attaques selon le jour, l'heure et la saison permet de compléter les principales analyses qui précèdent. La répartition du jour des attaques fait apparaître des pics les lundis et mercredis ainsi que les fins de semaines, sans doute liés à une augmentation du risque proportionnelle au nombre de pratiquants (Lemahieu et alii, 2013). L'heure des attaques est connue, au moins approximativement, pour 44 des 46 d'entre elles. Près de $80 \%$ des attaques (33 sur les 44 renseignées) se sont déroulées après $14 \mathrm{~h}$ et près de $40 \%$ après $17 \mathrm{~h}$ (ce qui correspond, en fonction des saisons à La Réunion, au coucher du soleil). Les attaques mortelles, contrairement à une opinion courante, interviennent davantage en journée 
( $80 \%)$, entre $07 \mathrm{~h}$ et $10 \mathrm{~h}$ ou entre $11 \mathrm{~h}$ et $14 \mathrm{~h} 30$, plutôt que le soir après $17 \mathrm{~h}(20 \%)$. La répartition mensuelle des attaques (illustration 6) est assez homogène sur l'année, hormis le mois de juillet, qui marque un pic important avec pas moins de 9 attaques dont 4 mortelles. Pour les attaques mortelles en revanche, la période d'avril à septembre (hiver austral) en concentre une part élevée (75\%). Une hypothèse est de considérer que la période de l'hiver austral est la plus propice aux sports de glisse, à la faveur des conditions de houle optimale; c'est aussi la plus risquée en raison de la turbidité élevée de l'eau (Lagabrielle et alii, 2012), qu'affectionne le requin bouledogue ${ }^{11}$ (Tirard, 2011), ainsi que des comportements des requins, qui sont en période de reproduction, en particulier dans le secteur de Saint-Gilles les Bains (Soria, 2014, 2015), avec une éventuelle sur-agressivité des mâles.

Illustration 6 - Répartition mensuelle des attaques depuis 1980

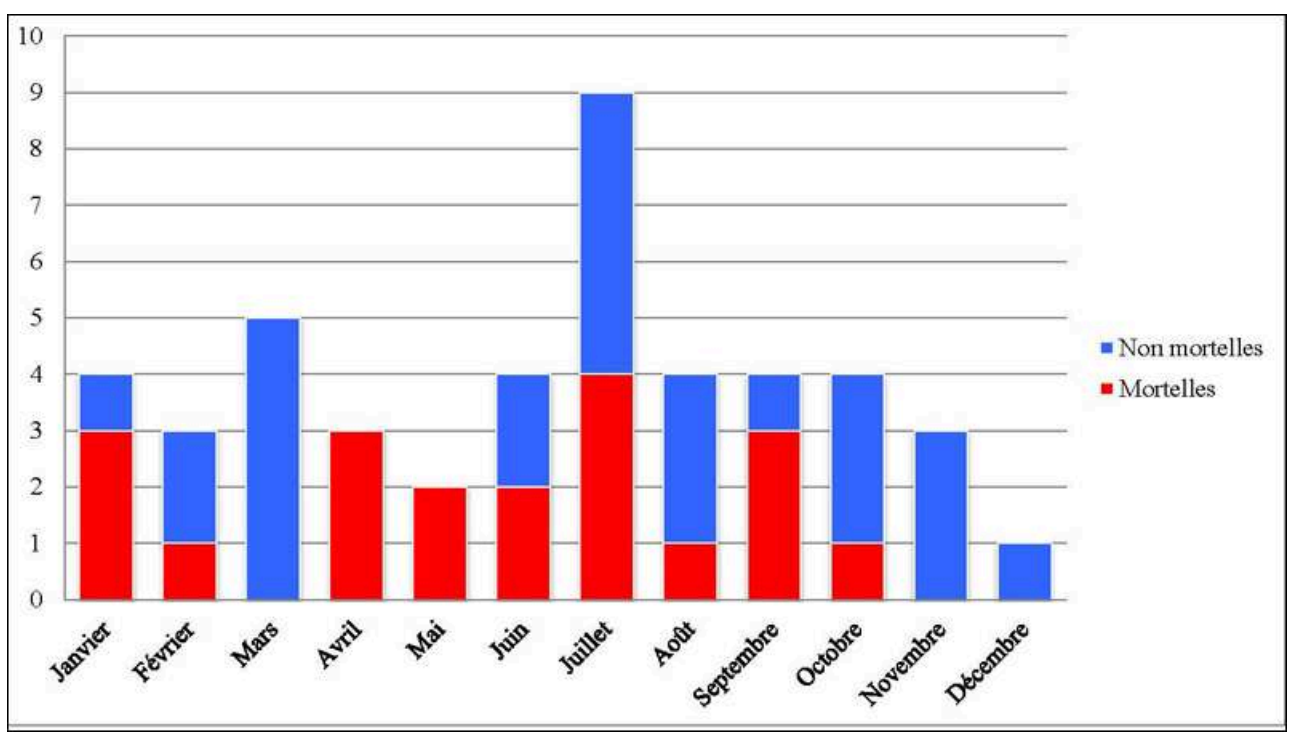

Sources : celles de l'illustration 2.

Ajoutons enfin une information issue de la littérature (Gauthier, 2012 et Lagabrielle et alii, 2012), selon laquelle deux tiers des attaques ont eu lieu en eaux troubles ou très troubles, ce qui est conforme aux observations dans d'autres territoires que La Réunion. En revanche, ici encore, contrairement à une idée reçue, environ $80 \%$ des attaques ont eu lieu alors que les jours précédents étaient ensoleillés et sans pluie.

Pour terminer, nous nous proposons de synthétiser quelques éléments du système explicatif des attaques de requin à La Réunion (illustration 7). Ces éléments, qui s'inscrivent dans trois champs (biotopique, biocénotique et anthropique), sont validés par des publications dans des revues scientifiques ${ }^{12}$ (cf. les sources). 
Illustration 7 - Quelques éléments explicatifs des attaques de requins à La Réunion selon la littérature

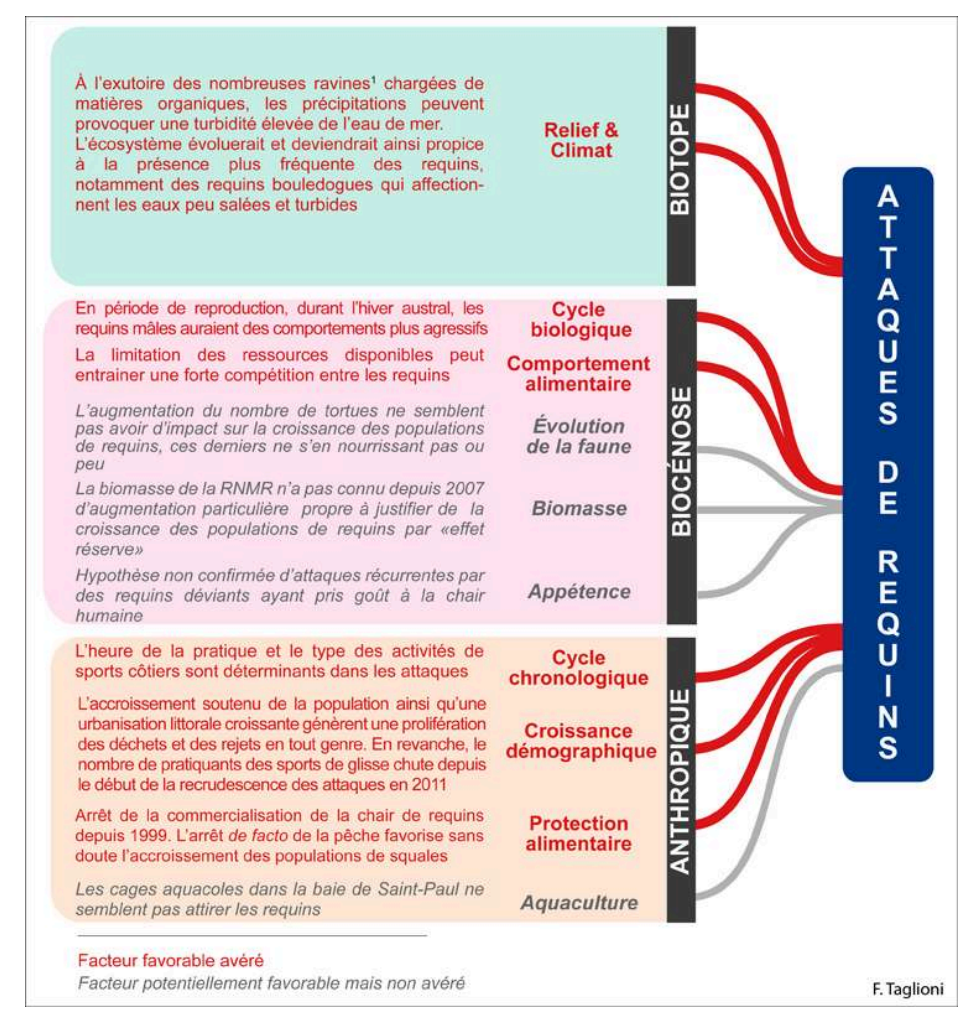

Note 1 : «Sur lî̂le de La Réunion, toutes les dépressions, profondes ou non, naturelles ou façonnées par l'homme (endiguées), et même les amorces de sillons qui découpent les pentes, sont qualifiées de ravines » (Bonnard, 2005).

Sources : Bigot et alii, 2014 ; Gauthier, 2012 ; Jean et alii, 2010 ; Lagabrielle et alii, 2012 ; Soria, 2012, 2014, 2015 ; Tézier, 2014.

Il n'apparaît pas de hiérarchie de nature à considérer ces éléments explicatifs isolément et à les appréhender analytiquement. Ils sont largement interdépendantes, perméables, et se combinent en produisant des interactions qui n'ont pas la même intensité selon les relations considérées. En l'état, cette figure nous rappelle qu'une attaque de requin est la résultante complexe de l'accumulation d'éléments humains, animaux et environnementaux. Il est donc très difficile de tendre vers un modèle explicatif et encore moins prédictif. D'autant que la relative étroitesse du nombre d'attaques des 35 dernières années à La Réunion $(n=48)$ ne permet pas en l'état actuel des connaissances scientifiques sur l'écologie des requins impliqués de conclure sur les causes profondes de la crise du requin à La Réunion depuis 2011. Néanmoins, les scientifiques de l'Institut de recherche pour le développement (IRD) mènent depuis 2011 un programme de recherche à La Réunion. Ce programme, sur lequel nous reviendrons dans la deuxième partie, est une action majeure et centrale des scientifiques pour faire progresser la connaissance de l'écologie et de l'habitat des deux principaux requins impliqués dans les attaques à La Réunion (Soria, 2015).

Ces premiers éléments d'analyses et de compréhension des attaques de requins à La Réunion nécessitent maintenant une mise en perspective avec le contexte institutionnel et politique de la gestion du risque requin dans l'île ces dernières années. 


\section{La gestion de la crise du requin}

\section{Mise en place de la Réserve naturelle marine : entre autorité et concertation}

La crise du requin s'installe en 2011 à la suite de l'attaque mortelle du 19 septembre dans un contexte institutionnel déjà générateur de conflits notamment avec la création, en 2007, de la Réserve naturelle marine de La Réunion (RNMR). De facto, cette attaque est véritablement celle ${ }^{13}$ qui, en raison de la personnalité du pratiquant et des conditions de l'attaque, installe une crise du requin dans la société réunionnaise. La RNMR sera rapidement désignée comme responsable de cette crise. En effet, elle cristallise des enjeux de pouvoir et donne l'état des relations que peuvent entretenir les acteurs environnementaux d'un côté et les acteurs économiques et sportifs de l'autre. Ces derniers ont subi la formalisation de la RNMR comme l'expression d'une gestion purement réglementaire et une remise en cause de leur légitimité. Les plus prompts à réagir sont souvent des acteurs sportifs emblématiques, dotés d'un certain pouvoir, de nature principalement symbolique. Cet état de fait s'est accompagné de nombreuses critiques de la gestion et du gestionnaire, dans une ambiance conflictuelle (Thomassin, 2011). Ce climat se retrouve dès le début de la crise du requin, avec des acteurs environnementaux pointés du doigt par certains pratiquants et des acteurs institutionnels qui, entre autorité et concertation, éprouvent des difficultés à se positionner.

Pour bien comprendre pourquoi la RNMR a été autant désignée comme la principale cause de l'augmentation des attaques de requins, il nous faut retracer le contexte institutionnel ainsi que l'articulation des jeux d'acteurs qui ont présidé à sa création. La décentralisation, génératrice de nouveaux mécanismes de gouvernance, a complexifié l'action des pouvoirs publics dans leur gestion des littoraux. Si le gouvernement implique les autorités politiques légitimes, en revanche la gouvernance s'inscrit dans une négociation entre acteurs concernés sous différentes formes : réseau, légitimité, pouvoir, contrats (Allemand, 2000). À La Réunion, l'articulation gouvernement/ gouvernance ne semble pas réussir à se mettre en place. C'est pourquoi, en juin 2013, l'État a mandaté un médiateur pour réguler les conflits d'usage dans la RNMR. Un rapport (Barret, 2013) a été rédigé par le médiateur qui estime « essentiel de renforcer le dialogue entre scientifiques et usagers, notamment les usagers qui ont accumulé de nombreuses observations au sein de la réserve ", essayant ainsi d'atténuer ce sentiment de relation descendante s'imposant aux usagers et de primauté des acteurs scientifiques. Une des explications de la difficile articulation gouvernement/ gouvernance tient pour Bernard Kalaora (2013) à la prégnance d'un modèle « culturaliste » dans la vision environnementaliste des Français. Selon lui, « La culture française n'a pas de concept d'environnement, elle en associe mal les registres contradictoires de proximité et de distance, de complexité technique et scientifique et de subjectivité, de connaissance et d'action. Elle n'en comprend pas la problématique ni les effets d'échelle». L'imaginaire français de protection de l'environnement, naturaliste et esthétique, est en décalage avec l'approche anglo-saxonne biologique et gestionnaire plus à même de répondre aux problématiques des pratiques sportives de nature dans un milieu fragile. Cette vision naturaliste et pittoresque de l'écologie, loin du pragmatisme anglo-saxon plus intéressé par la biodiversité et la régulation, 
témoigne également de la vision mythique de la mer qu'ont les Français. En effet, comme l'analyse Catherine Mougenot (2003), l'évolution actuelle de la protection de la nature implique que l'Homme soit non seulement présent dans la nature, mais en plus qu'il ait la charge de la gérer pour maintenir la biodiversité des espaces protégés. Cette gestion concertée, qui fait référence à une gouvernance territoriale des espaces naturels et au-delà du développement des sports côtiers, est rendue difficile par la vision culturaliste du littoral présente chez de nombreux acteurs (Kalaora, 2010). Ce positionnement rend difficile la territorialisation du littoral qui reste le pré carré des seuls conservateurs ou amoureux autoproclamés de la nature. Ce constat peut entraîner un questionnement autour de la gouvernance du littoral réunionnais et des enjeux de pouvoirs qui sous-tendent cette gestion (Guiltat, 2011). Ainsi, on note actuellement une ambiguïté des politiques environnementales, soulignée par Jacques Theys (2002). En effet, elles peuvent prendre la forme d'une gestion autoritaire et administrative (gouvernement) ou tenter d'établir une concertation et une gouvernance environnementale (gouvernance).

La création de la RNMR en 2007 a permis la mise en place d'un découpage de l'ensemble de la zone corallienne comprise entre Saint Paul et Étang-Salé (cf. illustration 2). Malgré les consultations préalables, ce zonage a été perçu, par les prestataires ${ }^{14}$ et les pratiquants de sports côtiers, comme une prise de pouvoir du gestionnaire du parc et de la Direction de l'environnement, de l'aménagement et du logement (DEAL; exDIREN) cherchant à les écarter du processus décisionnel (Guiltat, 2011; Thomassin, 2011). Prestataires et pratiquants ont subi la création de la RNMR comme une gestion purement réglementaire. La DEAL est d'ailleurs souvent prise pour cible de la vindicte des autres acteurs. Elle représente le pouvoir institutionnel de l'État, d'autant plus que les fonctionnaires y travaillant changent au gré des affectations. Cette remise en cause des pratiques par des acteurs récemment arrivés dans l'île, souvent de passage et dont le pouvoir ne tient qu'à leur fonction, sans qu'ils soient appuyés par une légitimité de terrain, a pu accentuer cette ambiance conflictuelle. De l'avis des pratiquants de sports côtiers et des prestataires, les services déconcentrés de l'État en charge du littoral se sont contentés de rester dans la sphère des relations verticales (communication, information, consultation) sans entrer dans une véritable concertation (Beuret, 2006). Ainsi, le cas de la RNMR semble aller à l'encontre de la tendance actuelle en termes de gestion environnementale «qui consiste à mettre de côté, au moins pour un temps de négociation, les prérogatives réglementaires pour établir des partenariats sur la base minimale d'un dialogue voire même d'une gestion concertée " (Mounet, 2007). Les acteurs environnementaux à La Réunion sont décrits comme « trop souvent cantonnés dans leurs domaines de compétence, ne créant pas les synergies nécessaires avec l'univers des loisirs sportifs de nature, les considérant trop souvent encore comme de simples divertissements plutôt que comme des vecteurs structurants des territoires " (Bessy, 2008). En effet, les intérêts particuliers des pratiquants d'une manière générale n'ont pas été traduits en intérêts communs par les acteurs environnementaux. En d'autres termes, les responsables de la mise en place de la réserve n'ont pas su formuler une «question, une interrogation, susceptible de produire la convergence des acteurs concernés » (Amblard et alii., 2005).

Pour autant, ces conflits d'intérêts entre les gestionnaires de la RNMR et les prestataires/pratiquants de sports côtiers ne sont pas réductibles à l'expression d'« égoïsmes locaux» [comme un syndrome NIMBY ${ }^{15}$ appliqué aux sports côtiers (Wheaton, 2007)]. La mise en réseau des acteurs dans la gestion environnementale et la 
mise en place de la réserve révèlent certaines difficultés et montrent une gouvernance déséquilibrée, avec des acteurs sportifs et économiques mal reconnus, des acteurs politiques locaux faibles laissant le champ libre à l'État pour aménager à la place des acteurs locaux. On constate ainsi que la fonction intrinsèque de la RNMR passe derrière son intégration dans le territoire réunionnais. De fait, une réserve naturelle ne peut pas être un projet de territoire en soi. En revanche, un projet de territoire peut inclure une réserve avec une gouvernance adéquate, mais cela n'a pas été le cas à La Réunion. En effet, cette territorialisation n'est possible qu'en présence d'un portage politique fort. Or, dans le cadre de la réserve, le positionnement des acteurs politiques et institutionnels n'a pas été clair dès le début (Guiltat, 2011).

31 Les prestataires et les pratiquants de sports côtiers ne s'opposent pas systématiquement aux actions publiques mais manifestent bien plutôt leur volonté de participer et de peser sur l'aménagement d'un territoire qui est aussi le leur. Cela souligne que «les autorités publiques ne sont plus les seules dépositaires de l'intérêt général»(Theiller et Audeguis, 2008). C'est dans ce contexte conflictuel et avec cet historique mouvementé que la gestion de la crise du requin a dû s'organiser.

\section{La crise du requin, une illustration d'un gouvernement par l'urgence}

Le risque requin à La Réunion n'est pas né en 2011. Néanmoins, ce risque ne s'était pas manifesté de façon aussi polémique dans le débat public lorsqu'il avait été appréhendé par les pouvoirs publics dans le passé. Pour mémoire, rappelons que les littoraux réunionnais ont connu, entre 1980 et 2011, 31 attaques de squales, dont 15 mortelles. Ainsi, en 1995, un projet d'aménagement visant à limiter le risque requin dans la commune de Saint-Paul qui concentre la majorité des usages balnéaires, consistait à sécuriser la baie éponyme, réputée être très fréquentée par les requins. Si ce projet fut rapidement écarté pour des raisons de politique locale, différentes hypothèses de sécurisation restèrent néanmoins envisagées ${ }^{16}$. Dans cette perspective, un rapport commandé à l'IFREMER (Roos et alii, 1997.) parut en $1997^{17}$, sans entraîner de prise de position politique de l'autorité territoriale saint-pauloise. Bien que le risque requin ait été clairement identifié comme un problème pour le développement touristique et économique de l'île dès le milieu des années 1990, force est de constater qu'aucune volonté politique ne permit d'apporter de solutions significatives en matière de prévention du risque. Ainsi, dix années se passèrent sans nouveaux projets autour de ce problème. Il fallut attendre 2008, pour qu'à nouveau se posa la question du risque requin sous l'impulsion des inquiétudes des Maîtres-Nageurs Sauveteurs (MNS) de la municipalité de Saint-Paul à propos de la recrudescence d'observations de requins aux abords immédiats des zones de baignade. La réponse des élus en place et sous couvert de la préfecture, tendit à minimiser ces observations de requins sur les plages de la commune de Saint-Paul. La réponse de la mairie de Saint-Paul fut la suivante : « Nous avons, à cette occasion, saisi de cette question, Monsieur le Sous-Préfet de St Paul qui nous a répondu que la mise en œuvre d'un plan d'alerte départemental serait susceptible d'entretenir une psychose du danger requin alors que le risque demeure faible et qu'il appartient au maire de prendre les mesures adéquates de police dans sa zone de responsabilité. Compte tenu de ce qui précède, nous vous informons que nous n'envisageons pas de prendre des mesures permanentes d'information du public face au risque requin. C'est ainsi qu'en l'absence d'information sur la présence de requin aux abords ou dans une zone de baignade surveillée à un moment donné, votre 
responsabilité ne pourra pas être engagée si la flamme verte est hissée. Dans l'hypothèse où la présence de squales vous serait signalée par un usager ou en cas de doute sérieux ou enfin en présence avérée de requins, il vous revient de hisser le drapeau rouge (...) » (note interne à la mairie de Saint-Paul, 22 décembre 2008 citée par la presse régionale réunionnaise en juillet 2013). Précisons qu'à l'époque aucune signalétique requins n'existait sur les plages et qu'il faudra attendre fin 2011 pour que cette dernière devienne effective.

La vulnérabilité au risque d'attaques de requins résulte d'incompatibilités spatiales entre les activités de sports côtiers et l'aléa. En effet, la présence accrue de requins le long de la côte devient un risque, car elle se superpose à un espace fortement anthropisé dans lequel se concentrent des pratiquants de sports côtiers. La production d'un territoire pertinent pour les usagers passe par la mise en place d'une gouvernance territoriale autour de la prise en compte du risque et de solutions acceptables pour le limiter. Dans les faits, à La Réunion, la seule solution proposée dès le début de la crise du requin a été l'interdiction des sports côtiers en dehors des lagons. Cette interdiction de facto a été ensuite formalisée par un arrêté préfectoral ${ }^{18}$. Cet arrêté d'interdiction, renouvelable tous les 6 mois, constitue la seule réponse concrète proposée par l'État, qui permet de contourner le problème en délaissant le territoire du risque de manière temporaire ou définitive (Tiberghien, 2008).

En parallèle à cette première réponse radicale, de multiples actions ont été envisagées pour enrayer la crise du requin qui se mesure notamment par son impact économique ${ }^{19}$. Nous allons discuter de celles qui ont été mises en œuvre ces derniers mois (Programme CHARC, vigie-requin, filets de protection, valorequins, diagnostic socio-économique de la crise du requin, caprequins).

La première des actions fut le lancement d'une étude scientifique sur l'écologie des requins. À ce propos, dans la gestion de crise, le rôle des sciences humaines est communément minoré. Elles interviennent généralement en aval des actions mises en place par les acteurs politiques, administratifs, économiques ou scientifiques des sciences dites «dures ». On retrouve cette priorisation durant la crise du requin, avec notamment la mise en place du programme « Connaissances de l'écologie et de l'habitat de deux espèces de requins côtiers sur la côte ouest de La Réunion ${ }^{20}$ » (CHARC). Bien que les autorités aient été promptes à lancer cette étude, un programme équivalent avait été présenté en 2008 sans être retenu. Ce programme aurait dû, à l'évidence, être initié depuis déjà de longues années en raison du manque pressant de connaissances sur l'écologie des requins de La Réunion. Néanmoins, et en dépit des premiers résultats très prometteurs, il ne peut en aucune façon constituer une réponse institutionnelle, et encore moins concrète, à la gestion de la crise en cours (Soria, 2015). C'est une des raisons de la perception négative de ce programme par certains usagers.

Les changements de direction dans les solutions politiques envisagées sont récurrents depuis le début de la crise et même avant que celle-ci ne débute. Ainsi, le dispositif vigie-requin déployé au cours de l'année 2012 a-t-il connu des errements. Ce dispositif consistait, d'une part, à «mesurer les conditions environnementales favorables aux activités de surf et, d'autre part, à mettre en place des équipes de surveillance et d'intervention sur et sous l'eau lorsque les activités encadrées étaient autorisées " (Info requin). Le 10 juillet 2014, Jean-Luc Marx, préfet de La Réunion, commentant l'étude autour du dispositif vigie-requin, fait état de 71 sorties au cours du premier semestre 2014, totalisant plus de 800 heures de vidéo sans aucune rencontre avec un 
squale. « Nous pouvons penser que lorsque l'homme occupe la colonne d'eau, l'animal craintif qu'est le requin ne s'y aventure pas ", assure-t-il ${ }^{21}$. Bien que présenté comme un dispositif innovant en 2012, il a fait l'objet d'une expertise négative en 2014, qui conclut à la faiblesse du protocole, pointant l'absence de référence établie aux plans national et international. Sous couvert de cette expertise, le nouveau préfet, nommé le 31 juillet 2014, a décidé de stopper le projet le 11 novembre 2014. Pourtant, dès le 14 novembre 2014, le président de Région, après avoir rencontré les acteurs des sports côtiers, s'est engagé à augmenter l'effort de la Région afin que soit amplifié très rapidement le programme vigie-requin. Ce dernier est une parfaite illustration des tâtonnements des gestionnaires.

Une autre solution évoquée au début de la crise est la mise en place de filets antirequins. Aussi, des filets provisoires ont-ils été déployés dès décembre 2011 pour les vacances de l'été austral. À l'époque, ce sont les maitres-nageurs sauveteurs (MNS) saint-paulois eux-mêmes qui avaient pris l'initiative de confectionner des filets de délimitation et de protection qui ont rapidement montré leurs limites en terme de pérennité. Pour des raisons techniques et météorologiques, ce premier filet a souvent été enlevé. La baignade restait cependant autorisée sous conditions avec une surveillance renforcée MNS et Patrouille, observation et alerte. Or, les conclusions scientifiques et la littérature spécifique, notamment sur le requin bouledogue, ne vont pas dans le sens de cette autorisation de baignade. Désormais et depuis octobre 2014, le nouveau filet déployé à Boucan Canot offre a priori une bonne protection tout en permettant une pratique limitée du surf à Boucan Canot. Ce dispositif devrait être déployé sur l'ensemble des spots de glisse de la commune de Saint-Paul dans le courant de l'année 2015.

L'étude valorequins ${ }^{22}$ porte sur l'évaluation de la toxicité éventuelle de la chair de requin. L'objectif annoncé par les autorités est de réintroduire le requin dans l'alimentation des Réunionnais ainsi qu'une valorisation sous forme de nourriture pour animaux et de produits de beauté. À l'inverse du programme CHARC, non létal pour les requins, cette étude ne fait pas l'unanimité et ses détracteurs, notamment les associations environnementalistes, y voient une manière hypocrite pour l'État d'autoriser les prélèvements. La récente décision de l'Agence nationale de sécurité sanitaire de l'alimentation, de l'environnement et du travail (ANSES) concernant le risque lié à la consommation des requins tigres et bouledogues à La Réunion illustre le positionnement ambigu de l'État. L'ANSES considère qu'il n'est pas possible d'écarter le risque de toxicité, même si aucune analyse à ce jour n'a mis en évidence la présence de ciguatoxines dans la chair des requins (ANSES, 2015). Contrairement à Mayotte, cette décision a pour conséquence l'interdiction de la commercialisation de la chair du requin depuis $1999^{23}$ et freine tout projet de valorisation, tout en permettant la poursuite du programme de pêche.

En août 2013, la DEAL lance un marché public de prestations intellectuelles nommé "Analyse de la crise requin à La Réunion dans les champs des sciences sociologiques, de l'information et de la communication et définition d'une stratégie de communication de crise et hors crise ${ }^{24}$. Les résultats de cette étude (Jaccoud, 2014) mettent en évidence les distances entre les attentes des usagers et la perception par ces derniers des décisions prises par les autorités. L'auteur va plus loin en assimilant la crise du requin à un révélateur des dysfonctionnements de la société réunionnaise. Les sciences humaines se voient ainsi attribuer un rôle à la fois précis dans ses objectifs 
pluridisciplinaires ${ }^{25}$ et restreint dans son périmètre d'application (faire de la communication). Nous espérons toutefois que la compréhension des conduites à risque et la gestion collective du risque en terme d'acceptabilité sociale, trop souvent opposées ou séparées, soient mises en application. En effet, l'efficacité d'une politique de gestion des risques dépend grandement de la part faite à l'analyse des représentations collectives, des rumeurs et des rapports de force (Mancebo, 2003) qui sont au cœur du débat depuis le début de crise du requin.

Quant à lui, le programme cap requins a été lancé en janvier 2014. Il est porté par le comité des pêches de La Réunion et vise à mieux comprendre la capturabilité des requins avec différents dispositifs de pêche (la drumline et la palangre de fond). Ces dispositifs prévoient soit la capture des plus gros spécimens de requins (utilisés ensuite dans le cadre de valorequins), soit le marquage des requins non ciblés. Fort des premiers retours, semble-t-il concluants, ce programme dit de sécurisation est présenté par ses défenseurs comme l'une des solutions possibles à cette crise. Il a également des détracteurs représentés par un collectif d'associations environnementales opposé à toute forme de prélèvement.

Le déploiement des solutions techniques envisagées que l'on vient de présenter laisse à penser qu'hésitations et tâtonnements ont présidé à la gestion de la crise du requin par les autorités compétentes (État et collectivités locales). Néanmoins, la création ex nihilo par la préfecture en 2012 du Comité réunionnais de réduction du risque requins (C4R) institutionnalise la gestion du risque requin. À cela s'ajoutent, en arrière-plan, des enjeux de pouvoirs et des problèmes de gouvernance. Il convient également de revenir sur le rôle joué par les pratiquants de sports côtiers et plus précisément les surfeurs, plus directement concernés par les attaques de requins. En effet, les pouvoirs locaux ont toujours été réticents à développer une véritable politique autour des sports côtiers et du surf en particulier. Pour Christophe Guibert (2006a), l'histoire du surf, aussi subjective et imposée soit-elle dans les magazines de surf et les discours marketing, offre une image déformée, presque mythique du surfeur, qui influence souvent négativement les élus locaux. De plus, à La Réunion, le surf et les sports côtiers en général sont perçus comme des pratiques métropolitaines importées et réservées à une frange aisée de la population (Thiann-Bo Morel, Duret, 2013; Jaccoud, 2014). Ces représentations vont se retrouver dans la place donnée au surf mais aussi aux autres sports côtiers par rapport à d'autres activités sportives plus traditionnelles. Les prises de position et les politiques sportives des élus ne relèvent donc pas exclusivement de savoirs et de pratiques économiquement et politiquement rationnelles. La culture des élus locaux et leur représentation classique du sport les poussent à se tourner principalement vers les associations sportives malgré le développement d'autres modalités de pratique. "Faire du sport au sein d'un club est considéré comme une affaire sérieuse dans la mesure où la participation associative demeure dans les représentations, aussi bien des dirigeants sportifs que des élus politiques, un lieu de socialisation, d'éducation et de citoyennetê » (Honta, 2010). Dans le cas de ces activités classiques, les représentations collectives sont fortement enracinées et les élus y perçoivent un intérêt électoraliste facilement identifiable et sur lequel ils peuvent influer. En revanche, pour le surf et les sports côtiers, les représentations sont beaucoup plus instables, voire contradictoires, le capital électoral semble moins évident, même si les élus peuvent avoir l'intuition que ces pratiques ont un rôle économique et social à jouer. 
42 La crise du requin exacerbe ces représentations et le positionnement des acteurs politiques. Les surfeurs et les pratiquants de sports côtiers (Guiltat, 2011), ne se sentant pas inscrits dans «l'agenda politique ${ }^{26} »$ (Padioleau, 1982) des élus en place, vont s'immiscer dans la récente campagne des élections municipales de mars 2014. À contrecourant des stéréotypes décrits par Guibert (2006a), c'est par le versant sportif et économique qu'ils vont se présenter et pour certains être élus. Les surfeurs entrent ainsi en politique. Si la parole n'est pas entendue hors du cercle politique et étatique, les surfeurs vont s'impliquer en politique. C'est ce qui s'est passé dans les villes de Saint-Paul et de Saint-Leu. Ainsi, durant les élections municipales de mars 2014, la crise du requin, sans être un enjeu majeur, a été au cœur de la campagne politique. À SaintLeu, Christophe Mulquin ${ }^{27}$ rejoint l'équipe du maire sortant Thierry Robert (élu au premier tour avec $72 \%$ des voix) en qualité de conseiller municipal en charge des activités nautiques et de la mer. À Saint-Paul, le changement est également important. La favorite des sondages, la députée-maire Huguette Bello, est battue par son adversaire Joseph Sinimalé qui avait mis la gestion de la crise du requin au centre de sa campagne. Au total, ce sont trois conseillers municipaux proches du monde du surf et des sports côtiers qui sont alors élus, dont Patrick Florès, entraîneur des équipes de France et père du champion du monde de surf Jeremy Flores. Ces dynamiques politiques à l'échelle locale s'inscrivent dans une inversion du rapport de force entre surfeurs et élus (Guibert, 2006b). Comme si la gravité de la crise du requin et les approximations dans sa gestion avaient contraint les pratiquants à passer de l'autre côté du miroir en s'invitant dans le jeu politique, pointant ainsi l'échec de la gouvernance en place.

On le constate, l'élaboration d'une politique publique doit être considérée comme la résultante d'interactions entre plusieurs acteurs, qu'ils soient sportifs, économiques, environnementaux ou encore aménageurs. Ce nouveau mode de relations, fondé sur la complexité et la multiplicité, est à l'origine de l'ouverture du « jeu collectif » (Honta, 2010) où les politiques publiques du sport sortent des services administratifs, se contractualisent et se partagent entre les différents acteurs. Cette transition s'apparente à un glissement d'un gouvernement municipal ${ }^{28}$ vers une forme de gouvernance territoriale. Cette dernière étant un ensemble de dispositifs et de procédures ne fonctionnant que s'ils sont appropriés, mobilisés par des acteurs ayant un accès équitable à la décision, partageant un minimum d'intérêts communs, et disposant de marges de manœuvre suffisantes. Dans le cas de la gestion de la crise du requin à La Réunion, à la place d'une gouvernance territoriale installée dans le temps et passant par un appui politique fort, nous avons assisté à une succession de réactions à chaud qui sont l'illustration d'un gouvernement agissant dans l'urgence plutôt que dans l'anticipation. Certes, il n'y a pas de manière simple pour gérer des attaques de requin. Néanmoins, la prise en compte précoce du problème est un élément primordial. Par exemple, un pays comme l'Australie se préoccupe du risque requin et de ses conséquences depuis plus d'un siècle (Neff, 2012 ; Neff, Hueter, 2013). On peut citer également l'Afrique du Sud et son KwaZulu-Natal Sharks Board Maritime Centre of Excellence qui existe depuis plus de cinquante ans avec pour mission la protection des usagers de la mer face au risque requin, mais également une approche environnementale et touristique. Il sera nécessaire dans une prochaine étude d'apporter des exemples de gestion dans d'autres espaces dans le monde exposés au risque d'attaques de requins. 


\section{Conclusion}

44 Le risque d'attaques de requins est présent sur toutes les mers du globe et tend globalement à augmenter depuis quelques décennies. Néanmoins, c'est à La Réunion que le nombre d'attaques par kilomètre de linéaire côtier est sans doute l'un des plus élevé au monde, notamment depuis 2011. À l'analyse de la répartition spatiale des cas, on note une très forte dissymétrie est/ouest. Ainsi, la zone occidentale de l'île a été le théâtre, entre 2011 et avril 2015, de 17 attaques dont 7 mortelles. L'analyse de la carte interactive, construite à partir d'une base de données des 46 attaques depuis 1980, permet d'identifier plusieurs variables qui pourraient conditionner la survenue des attaques. Les paramètres qui semblent pertinents sont assez nombreux et le système est d'autant plus complexe à analyser que le nombre d'attaques recensées reste trop limité pour que les interprétations soient hautement significatives et les prévisions possibles. Les interactions entre l'homme et le requin restent donc un sujet de recherches scientifiques qui nécessitent des approfondissements dont certains sont en cours de réalisation.

On le sait, les politiques environnementales se divisent en deux catégories pouvant soit prendre une forme plutôt administrative et autoritaire, soit tenter d'établir une vraie concertation couplée à une gouvernance partagée. Dans le cas de la Réserve naturelle marine, entre autorité et concertation, les acteurs institutionnels n'ont pas clairement réussi à se positionner. En effet, depuis sa mise en place jusqu'à la veille de la crise du requin, la gestion de la réserve naturelle marine s'illustre par une relation difficile entre les usagers et les gestionnaires. La politique au coup par coup adoptée par les autorités dans cette crise du requin semble en décalage avec une approche anglosaxonne biologique et gestionnaire. Une telle approche aurait sans doute été la mieux à même de répondre à la cohabitation entre sports côtiers, préservation du milieu et risque dans un climat moins passionnel et plus responsable. On peut ainsi penser que si ce risque requin avait été appréhendé auparavant, dès les années 1990, par les autorités compétentes, cette crise n'aurait pas pris une telle ampleur et n'aurait pas hypothéqué le développement des sports côtiers pour les années à venir. En l'état, La Réunion devient un laboratoire à ciel ouvert d'expérimentations de solutions techniques afin de minimiser le risque requin. Néanmoins, l'actualité est têtue et les attaques continuent à se succéder en remettant en question la notion même de crise qui par définition ne s'inscrit pas, a priori, dans le temps long.

\section{BIBLIOGRAPHIE}

Allemand S., 2000. Gouvernance: le pouvoir partagé. Sciences humaines, nº 101, p. 12-18

Amblard H., Bernoux P., Herreros G., Livian, Y. , 2005. Les nouvelles approches sociologiques des organisations. Paris, Seuil, 300 p. 
ANSES, 2015. Contamination des requins, notamment tigre et bouledogue, par des ciguatoxines : occurrence, méthodes analytiques, cas humains rapportés et éléments d'éthologie. Maison Alfort, Rapport d'expertise collective, $97 \mathrm{p}$. https://www.anses.fr/sites/default/files/documents/ ERCA2013sa0198Ra.pdf

Barret P., 2013. Réguler les conflits d'usage dans la réserve naturelle marine de La Réunion. Est-il possible de mettre en place une médiation et à quelles conditions ? Rapport interne, Société DialTer, $13 \mathrm{p}$.

Bessy O., Naria, O., 2004. Loisirs et tourisme sportif de nature à La Réunion. État des lieux, enjeux et perspectives en matière de développement durable. Saint-Denis, CCEE-Réunion, $130 \mathrm{p}$.

Bessy O., 2010. Marathons, trails, raids et développement territorial. Revue Espace, $n^{\circ} 287$, p. 10-19. Beuret J., 2006. La conduite de la concertation: Pour la gestion de l'environnement et le partage des ressources. Paris, L'Harmattan, $341 \mathrm{p}$.

Bigot L., Bruggemann H., Cadet C., Chabanet P., Durville P., Guillaume M., Hoarau L., Mulochau T., Penin L., 2014. Point 1 du suivi de l'effet réserve sur les communautés ichtyologiques et benthiques récifales - secteurs de La Saline et de Saint Leu. Rapport ECOMAR, IRD, MNHN, 52 p.

Bonnard S., 2005. Caractérisation typologique des ravines «non-pérennes » à forte valeur environnementale de l'île de La Réunion. Mémoire de DESS Sciences et Gestion de l'Environnement Tropical, Université de La Réunion, 198 p.

Burgess G., Callahan, 1996. Worldwide Patterns of White Shark Attacks on Humans. In Klimley A., Ainley D. (dir.), Great White Sharks. The biology of Carcharodon carcharias. San Diego, Academic Press, p. $457-469$

Curmi A., 2005. Taking a bite out of fiction-Media effects and social fears. A case study on « Jaws ». Malta, University of Malta. $71 \mathrm{p}$.

Fabing, P., 2014. Impact économique de la crise requin à la Réunion. Saint-Denis de La Réunion, SAGIS/ DEAL, 50 p. http://www.info-requin.re/IMG/pdf/

Impact_economique_crise_requin_a_la_Reunion_-_Sagis_-_Rapport_final_novembre_2014.pdf

FAO, 2014. La situation mondiale des pêches et de l'aquaculture. Rome, Organisation des Nations unies pour l'alimentation et l'agriculture, $255 \mathrm{p}$.

Gauthier C., 2012. Expertise médicale des victimes d'attaques de requins à l'île de La Réunion. Thèse de médecine, Université de Bordeaux II, 264 p.

Guiltat S., 2011. Le rôle des sports côtiers dans le développement territorial de l'Ouest de La Réunion: entre nouvelle maritimité et jeux d'acteurs. Thèse de doctorat de l'Université de La Réunion, $321 \mathrm{p}$. Gazzo, G., Mességué F., Maillaud C., Esclapez J-B., Rémy J-C., Passe J., van Grevelynghe G., 2000. Grand Livre de la Chasse Sous-Marine, Saint-Denis, Orphie, $432 \mathrm{p}$.

Guibert C., 2006a. Politiques de communication et identifications territoriales différenciées: les usages politiques des vagues et de l'univers du surf par les municipalités de la côte Aquitaine. Téoros. Revue de recherche en tourisme, $n^{\circ} 2, p .62-71$.

Guibert C., 2006b. L'univers du surf et stratégies politiques en Aquitaine. Paris, L' Harmattan, 321 p. Haschar- Noé N., 2005. Sport et «identité locale » : l'exemple de deux projets d'aménagements sportifs et touristiques intercommunaux. In Bouchet, P., Sobry, C. (dir.), Management et marketing du sport : du local au global. Paris, Septentrion, 199 p. 
Hoffer O., 2013. Quand le littoral se ferme. Quelle gouvernance de l'accès et des usages de l'interface littorale dans les agglomérations de Nouméa, Auckland et Port-Vila? Thèse de doctorat, Université de La Réunion, $418 \mathrm{p}$.

Honta M., 2010. Gouverner le sport: action publique et territoire. Grenoble, Presses Universitaires de Grenoble, 200 p.

Human to human, 2014. Audit des conversations Web, période de veille été 2012- février 2014. Paris, Human to human, 47 p. http://www.info-requin.re/IMG/pdf/Audit_Digital.pdf

Ipsos, 2014. Analyse de la crise requin. Enquête quantitative auprès de la population réunionnaise. SaintDenis de La Réunion, Ipsos, 125 p. http://www.info-requin.re/IMG/pdf/Ipsos_-

_Rapport_Crise_Requin_.pdf

Jaccoud A., 2014. Mieux comprendre pour mieux agir. Approche sociale de la crise requin. Saint-Denis de La Réunion, DEAL, 172 p. http://www.info-requin.re/IMG/pdf/Etude_socio_-RAPPORT_DEAL__Approche_sociale_de_la_crise_requin_-.pdf

Jaquemet S., Smale M., Blaison A., Guyomard D., Soria M., 2012. First observation of a pregnant Tiger shark (Galeocerdo cuvier) at Reunion island, Western Indian Ocean. Western Indian Ocean Journal of Marine Science, vol. 11, p. 205-207.

Jean C., Cicionne S., Ballorain K., Georges, J-Y., Bourjea J., 2010. Ultralight aircrafyt surveys reveal marine turtle population increases along the west coast of Reunion Island. Oryx, vol. $44, \mathrm{n}^{\circ} 2$, p. 223-229.

Jobert A., 1998. L'aménagement en politique. Ou ce que le syndrome NIMBY nous dit de l'intérêt général. Politix, nº 42, p. 67-92.

Kalaora, B., 2010. Rivages en devenir : des horizons pour le conservatoire du littoral. Paris, La Documentation française, $280 \mathrm{p}$.

Kalaora, B., Vlassopoulos C., 2013. Pour une sociologie de l'environnement. Environnement, société et politique. Paris, Champ Vallon, 301 p.

Lagabrielle E., Loiseau N., Verlinden N., Chabanet P., Soria M., 2012. Analyse des conditions environnementales et des usages de la mer associés aux attaques de requin à l'île de la Réunion entre 1980 et 2011. Rapport non publié, Institut de recherche pour le développement (IRD), Saint-Denis de La Réunion, $34 \mathrm{p}$.

Lemahieu A., Pennober G., David G., Lavigne F., Pothin K., Gérard L., 2013. Elaboration d'un protocole de suivi de la fréquentation au sein de la réserve naturelle marine de La Réunion. VertigO, vol. 13, n 1.http://vertigo.revues.org/13379

Libaert T., 2009. Crises et facteur humain. Bruxelles, De Boeck Supérieur, 272 p.

Mancebo F., 2003. Questions d'environnement pour l'environnement et l'urbanisme. Nantes, Editions du Temps, $287 \mathrm{p}$.

Metzger P., D'Ercole R., 2011. Les risques en milieu urbain : éléments de réflexion. EchoGéo, $\mathrm{n}^{\circ} 18$, http://echogeo.revues.org/12640

Mougenot C., 2003. Prendre soin de la nature ordinaire. Bordeaux, MSH INRA, 230 p.

Mounet J-P., 2007. La gestion environnementale des sports de nature: entre laisser-faire, autorité et concertation. Développement durable et territoires, varia. http:// developpementdurable.revues.org/3817 
Neff C., 2012. Australian Beach Safety and the Politics of Shark Attacks. Coastal Management, vol. $40, \mathrm{n}^{\circ} 1$, p. 88-106.

Neff C., Hueter, R., 2013. Science, policy, and the public discourse of shark "attack": a proposal for reclassifying human-shark interactions. Journal of environmental studies and sciences, $\mathrm{n}^{\circ} 3$, p. 65-73.

Neff C., 2014. The Jaws effect : how movie narratives are used to influence policy responses to shark bites in Western Australia. Australian journal of political science, p. 114-127.

Padioleau J., 1982. L'Etat au concret. Paris, Presses universitaires de France, 222 p.

Rard M., Menou A., 2011. Bilan sur les activités nautiques à l'île de La Réunion. Saint-Denis, Observatoire marin de La Réunion, 80 p. http://omar.fr/wp-content/uploads/2014/05/ RardEtMenou2011-RapportActivit\%C3\%A9sNautiquesPr\%C3\%A9fecture.pdf

Rard M., 2013. Inventaire Réunionnais des attaques de requins. La Réunion, Observatoire marin de La Réunion, 24 p. http://omar.fr/wp-content/uploads/2013/10/IRAR-

InventaireReunionAttaqueRequins-OMAR2013.10.27.pdf

Robbins W., Peddemors V., Kennelly S., Ives M., 2014. Experimental evaluation of shark detection rates by aerial observers. PLoS One, vol. $9, \mathrm{n}^{\circ} 2 . \mathrm{http}$ //www.plosone.org/article/

info\%3Adoi\%2F10.1371\%2Fjournal.pone.0083456

Roinsard N., 2014. Pauvreté et inégalités de classe à La Réunion. Etudes rurale, n 194, p. 173-189.

Roos D., Van der Elst R., Guyomard D., René, F., 1997. Proposition pour la mise en place d'un programme contribuant à la gestion du risque requin dans le cadre de l'aménagement d'une aire de baignade dans la baie de St-Paul. Le Port, IFREMER, $50 \mathrm{p}$.

Roux M., 1997. L'imaginaire marin des Français : mythe et géographie de la mer. Paris, L'Harmattan, $220 \mathrm{p}$.

Soria, M. (coordonnateur), 2012. Programme CHARC (Connaissances de l'écologie et de l'HAbitat de deux espèces de Requins Côtiers sur la côte Ouest de la Réunion). Rapport non publié, Institut de recherche pour le développement (IRD), Saint-Denis de La Réunion, 34 p. http://www.la-reunion.ird.fr/ content/download/61646/479375/version/1/file/Programme+CHARC.pdf

Soria M. (coordonnateur), 2014. Bilan de l'analyse des données de marquage collectées du mois de décembre 2011 au mois de septembre 2013 dans le cadre du programme CHARC. Rapport non publié, Institut de recherche pour le développement (IRD), Saint-Denis de La Réunion, 31 p. http:// www.la-reunion.ird.fr/content/download/78759/599945/version/1/file/

Bilan+de+l\%27analyse+des+donn\%C3\%A9es+de+marquage+collect\%C3\%A9es+de+d\%C3\%A9cembre+2011+ \%C3\%A0+septembre+2013.pdf

Soria M. (coordonnateur), 2015. Rapport scientifique final du programme CHARC (Connaissances de l'écologie et de l'HAbitat de deux espèces de Requins Côtiers sur la côte Ouest de la Réunion). Rapport non publié, Institut de recherche pour le développement (IRD), Saint-Denis de La Réunion, 132 p.

Surmont, E., 2014. La crise requin à La Réunion, entre science et fiction. Mémoire de master 1, ENS Lyon, $123 \mathrm{p}$.

Taglioni F., Dehecq J-S., 2009. L'environnement socio-spatial comme facteur d'émergence des maladies infectieuses. Le chikungunya dans l'océan Indien. Echogéo, $\mathrm{n}^{\circ} 9$, http:// echogeo.revues.org/index11168.html

Theiller D., Audeguis V., 2008. L'enjeu de développement durable des sports de nature estivaux dans la chaîne pyrénéenne. In Bessy O, Sport, loisir, tourisme et développement durable des territoires. Voiron, Presses Universitaires du Sport, p. 86-99. 
Thiann-Bo Morel M., Duret,P., 2013. Le risque requin, mise en risque de la pratique du surf à la Réunion. STAPS, $n^{\circ}$ 99, p. 23-36.

Thomassin A., 2011. "Des réserves sous réserve". Acceptation sociale des Aires Marines Protégées. L'exemple de la région sud-ouest de l'océan Indien. Thèse de doctorat, Université de La Réunion, 568 p. Tirard P., 2011. Requins du caillou. Nouméa, Edition Philippe Tirard, 271 p.

Van Grevelynghe G., Diringer A., Séret B., 1999. Tous les requins du monde : 300 espèces des mers du globe, Paris, Delachaux \& Niestlé, 336 p.

Weltz K., Kock A., Winker H., Attwood C., Sikweyiya M., 2013. The influence of environmental variables on the presence of white sharks, Carcharodon carcharias at two popular Cape Town bathing beaches: a generalized additive mixed model. PLoS One, vol. 8, $\mathrm{n}^{\circ} 7 \mathrm{http}: / /$ www.plosone.org/article/info\%3Adoi\%2F10.1371\%2Fjournal.pone.0068554

Werbrouck A., Van Grevelynghe G., Landron F., Charlier P., Loire C., Gauthier, C., 2014. Expertise médicolégale des victimes d'attaques et de morsures de requins à La Réunion. La Revue de Médecine Légale, $\mathrm{n}^{\circ} 142$, p. 110-121.

Wheaton B., 2007. Identity, politics, and the beach: environmental activism in Surfers Against Sewage. Leisure Studies, ${ }^{\circ} 3$, p. 279-302.

Worm B., Davis, B., Kettemer L., Ward-Paige C., Chapman, D., Heithaus M., Kessel S., Gruber S., 2013. Global catches, exploitation rates, and rebuilding options for sharks. Marine Policy, $\mathrm{n}^{\circ} 40$, p. 194-204.

\section{Webographie}

Info requin, préfecture de La Réunion http://www.info-requin.re/

Océan prévention Réunion http://www.opr.re/

Observatoire marin de La Réunion http://omar.fr/

Shark Attacks and Related Incidents File http://sharkattackfile.info

Squal'idees http://www.squalidees.com/

\section{Filmographie}

La Réunion en proie aux attaques de requins, 2011. M6, « Zone interdite », diffusé le 02/10/2011, 66 minutes.

Lecomte C., Potocki R., Millet S., 2014. L'île aux requins. France 2, "Envoyé spécial » (Les carnets de voyage), diffusé le 07/08/2014, 34 minutes

Crise requin à la Réunion, 2014. France 3, « Thalassa », diffusé le 16/05/2014, 21 minutes.

Leonard P., 2012. Pourquoi les requins attaquent ? FR3, « C'est pas sorcier », diffusé le 08/12/2012, 26 minutes.

Poirier P., 2014. Le requin, l'île et les surfeurs. France Ô, diffusé le 20/04/2014, 51 minutes

Tézier R., 2014. Attaques de requins à La Réunion : l'enquête. Réunion 1er, diffusé le 04/06/2014, 96 minutes.

Tézier R., 2015. Connaissances de l'écologie et de l'habitat de deux espèces de Requins côtiers. Film coproduit par l'IRD et Tec Tec Production, 21 minutes. http://www.la-reunion.ird.fr/toute-l- 
actualite/l-actualite/l-actualite-du-programme-charc/film-les-principaux-resultats-duprogramme-charc

\section{NOTES}

1. http://sharkattacksurvivors.com. Il existe également deux autres bases internationales avec des définitions différentes d'une attaque et une moindre exhaustivité que la première. Il s'agit de l'International Shark Attack File (ISAF) et du Global Shark Attack File (GSAF).

2. Shark attack file info, http://sharkattackfile.info

3. Nous remercions ici les auteurs des sources qui apparaissent sous l'illustration 2.

4. Les bichiques désignent deux types d'alevins de poissons que l'on pêche à La Réunion à l'embouchure des rivières, ainsi qu'en eau douce.

5. Les spots sont des lieux particulièrement favorables à la pratique d'un sport de nature, plus particulièrement un sport de glisse.

6. La Réserve naturelle marine de La Réunion (RNMR (illustration 2) a été créée en février 2007. Elle s'étend du Cap la Houssaye (commune de Saint-Paul) à la Roche aux oiseaux (commune de l'Étang Salé), soit sur $40 \mathrm{~km}$ de linéaire côtier.

7. Le ratio des attaques pour $100 \mathrm{~km}$ de linaire côtier serait encore plus élevé si l'on considérait que la RNMR comprend un lagon, portion non négligeable des $40 \mathrm{~km}$ du linéaire côtier, peu propice aux sports côtiers à l'exception de la nage.

8. On peut néanmoins tempérer ce constat en précisant que nous n'avons pas effectué le même mode de calcul, qui consiste à réduire le linéaire côtier à l'unique zone de pratiquants de sports côtiers, pour les autres zones d'attaques de requins dans le monde comme la Floride, Hawaï, l'Australie ou la République d'Afrique du Sud.

9. De ce point de vue, le risque d'attaques de requins est d'autant plus sensible que les victimes sont jeunes. En comparaison, le chikungunya, épidémie qui a frappé La Réunion entre 2005 et 2006 (Taglioni, Dehecq, 2009) a fait 220 victimes, soit 10 fois plus que les attaques mortelles de requins depuis 1980 ; mais les victimes étaient majoritairement des personnes âgées présentant déjà de lourdes pathologies.

10. Source : http://sharkattackfile.info

11. Le requin bouledogue est sans doute le premier responsable des attaques à La Réunion

12. Cependant, d'autres pistes, non encore scientifiquement validées, sont à explorer, comme par exemple les contraintes réglementaires imposées par la RNMR, qui limitent la présence de l'homme dans le milieu marin en ayant pour corollaire le non effarouchement des requins côtiers.

13. Elle fait néanmoins suite à 4 attaques la même année dont une mortelle (cf. illustration 2)

14. Nous désignons ici par le terme de "prestataires" les acteurs économiques liés aux sports côtiers.

15. NIMBY : Le syndrome NIMBY (Not In My Back Yard) peut se définir comme une attitude d'opposition d'acteurs locaux vis-à-vis d'un projet lorsque celui-ci est susceptible de comporter certaines nuisances ou modifications, réelles ou supposées, de leurs habitudes ou de leur cadre de vie. Dans le domaine du surf, on peut se reporter à la lecture de l'article de Belinda Wheaton sur l'activisme des surfeurs britanniques (Wheaton, 2007).

16. Comme par exemple, des filets discontinus (pour réduire le coût de l'entretien) très proches de la plage et sur une partie réduite de la baie, ou encore la construction de bassins artificiels. Cette solution est déjà utilisée sur les littoraux sud-africains et australiens depuis les années 1980.

17. Ce rapport tenant plus de la revue de littérature sur les dispositifs anti-requin existant, met l'accent sur le côté permanent et coûteux de telles mesures. En effet, une collectivité s'engageant 
dans ce type de sécurisation ne pourrait faire marche arrière pour des raisons économiques et de responsabilité.

18. Le 26 juillet 2013, devant la recrudescence des attaques de requins, le préfet de La Réunion a pris un arrêté interdisant certaines activités nautiques sur une partie du littoral de La Réunion. Dans son ordonnance du 13 août 2013, le juge des référés du Conseil d'État a constaté que «à court terme, seules les mesures d'interdiction de baignade et d'activités nautiques sont susceptibles de supprimer le risque d'attaques, à la condition que ces interdictions soient respectées ». Depuis cette date et en dépit de cette réglementation spécifique, quatre nouvelles attaques sont malheureusement à déplorer : le 26 octobre 2013 à l'Étang-Salé, le 22 juillet 2014 à Saint-Leu, le 14 février 2015 à l'Étang-Salé et le 12 avril 2015 à Boucan Canot.

19. Les pertes économiques se chiffrent à environ 33 millions d'euros depuis 2011 ; sans compter les pertes indirectes liées à la chute de fréquentations de la station balnéaire (Fabing, 2014 ; Jaccoud, 2014).

20. Le programme CHARC, initié en fin 2011, est un programme scientifique, confié aux chercheurs de l'IRD, financé par l'Europe (Fonds européen de développement régional, FEDER), la Région Réunion et l'État (DEAL) qui a pour but d'établir un état initial des connaissances sur l'écologie et sur l'habitat de deux des espèces de requins côtiers sur la côte Ouest de La Réunion (le requin tigre (Galeocerdo cuvier) et le requin bouledogue (Carcharhinus leucas) impliqués dans les attaques.

21. Interview publiée sur le site Imaz Pres Réunion (www.ipreunion.com/) le 10 juillet 2014

22. Dans un premier temps, cette étude, commencée en août 2012, fut baptisée "Ciguatera ». Le programme "Ciguatera 2 », rebaptisé "valorequin", doit s'appuyer à terme sur l'analyse de 90 requins prélevés ( 45 bouledogues et 45 tigres).

23. Cette décision a été motivée par une intoxication par des ciguatoxines en 1993 à La Réunion et également par de mortelles et répétitives intoxications dans l'État voisin de Madagascar. Alors qu'à Mayotte, département français, pourtant plus proche de Madagascar, aucune interdiction de commercialisation n'est en vigueur jusqu'à la déclaration de la ministre des outre-mer du 24 avril 2015

24. Cette commande se décline suivant plusieurs axes : sociologie, communication et économie. Elle a donné lieu à plusieurs études (Jaccoud, 2014 ; Ipsos, 2014 ; Fabing, 2014 ; Human to human, 2014).

25. Il s'agit de produire des analyses sur la population cible, comprendre ses perceptions ou les raisons de son apparente irrationalité. La question de l'acceptabilité du risque est centrale pour ce type de travaux.

26. Le concept issu des sciences politiques s'applique à l'ensemble « des problèmes perçus comme appelant un débat public, voire l'intervention des autorités publiques légitimes».

27. Christophe Mulquin a été coach des équipes de France minimes, cadets et ondines. Entraîneur et coach du Team Réunion depuis 18 ans, il a gagné, durant cette période, près de 50 titres de champion de France, plusieurs titres européens, et un titre de Champion du monde (International surfing association).

28. Municipal, mais également départemental ou régional selon l'échelon territorial sélectionné. 


\section{RÉSUMÉS}

La Réunion est particulièrement exposée au risque d'attaques de requins, globalement très supérieur à celui que connaissent d'autres territoires concernés dans le monde notamment en terme de mortalité. D'une part, cet article propose une analyse de la base de données sur les 46 attaques répertoriées entre 1980 et 2014. À partir de l'élaboration d'une carte interactive des attaques, la seule produite à ce jour sur le sujet, plusieurs variables explicatives qui pourraient conditionner la survenue des attaques sont identifiées et analysées. D'autre part, cette recherche est une réflexion sur la gestion institutionnelle de la crise du requin qui affecte La Réunion depuis 2011. La création et le fonctionnement de la Réserve naturelle marine de La Réunion (RNMR), sous un mode perçu comme administratif et autoritaire par une frange de la population va cristalliser une crise du requin à partir de 2011. Une succession de réactions à chaud par les autorités compétentes dans la gestion de cette crise témoigne d'un gouvernement par l'urgence en lieu et place d'une gouvernance territoriale moins passionnelle et mieux anticipée, qui aurait sans doute pu limiter l'ampleur et les conséquences de cette crise.

Reunion island is particularly exposed to shark attacks which have been more frequent than in any other area in the world. This article objectives are twofold. On the one hand it offers an analysis of the statistics of the 46 shark attacks between 1980 and 2014. Using the only interactive map created of these attacks to this day, several explicative variables that could condition the upsurge of attacks have been identified and analysed. On the other hand, this research analyses the institutional management of the shark crisis affecting Reunion Island since 2011. The creation of a natural marine reserve perceived as administrative and authoritarian crystallizes the shark attacks crises since 2011. A succession of on the spot reactions in the management of this crisis will illustrate that the government had an urgent management of the situation instead and in the place of a less passionate and more anticipated territorial governance which could doubtlessly have limited the magnitude and consequences of such a crisis.

\section{INDEX}

Mots-clés : Ile de La Réunion, réserve naturelle marine, risque requin, gestion du risque, gouvernance territoriale, sports côtiers

Keywords : Reunion Island, Natural marine area, risk of shark attack, risk management, territorial governance, coastal sports

\section{AUTEURS}

\section{FRANÇOIS TAGLIONI}

François Taglioni, francois.taglioni@univ-reunion.fr, est Professeur des universités à l'Université de La Réunion, membre de l'UMR PRODIG et du CREGUR. Spécialiste des petits espaces insulaires en développement dans le monde, il travaille sur les interactions santé/environnement dans le Pacifique et l'océan Indien ; site Internet de l'auteur : http://www.taglioni.net 


\section{SÉBASTIEN GUILTAT}

Sébastien Guiltat, sebastien.guiltat@univ-reunion.fr, est docteur en STAPS de l'Université de La Réunion où il est chargé de cours et membre de laboratoire IRISSE. Il est directeur de l'office municipal des sports de Saint-Paul (La Réunion) 